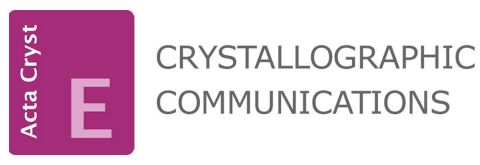

ISSN 2056-9890

Received 26 September 2015

Accepted 2 October 2015

Edited by A. J. Lough, University of Toronto, Canada

Keywords: crystal structure; 4,4'-bipyridyl; 4 alkoxybenzoic acid; hydrogen-bonded liquid crystal

CCDC references: 1429203; 1429204; 1429205

Supporting information: this article has supporting information at journals.iucr.org/e

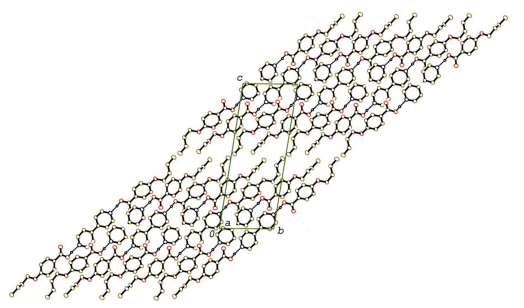

\section{Crystal structures of three co-crystals of 4,4'-bipyridyl with 4-alkoxybenzoic acids: 4-ethoxybenzoic acid-4,4'-bipyridyl (2/1), 4-n-propoxybenzoic acid-4,4'-bipyridyl (2/1) and 4-n-butoxybenzoic acid-4,4'-bipyridyl (2/1)}

\author{
Yohei Tabuchi, Kazuma Gotoh and Hiroyuki Ishida*
}

Department of Chemistry, Faculty of Science, Okayama University, Okayama 700-8530, Japan. *Correspondence e-mail: ishidah@cc.okayama-u.ac.jp

The crystal structures of three hydrogen-bonded co-crystals of 4-alkoxybenzoic acid-4,4'-bipyridyl (2/1), namely, $2 \mathrm{C}_{9} \mathrm{H}_{10} \mathrm{O}_{3} \cdot \mathrm{C}_{10} \mathrm{H}_{8} \mathrm{~N}_{2}$, (I), $2 \mathrm{C}_{10} \mathrm{H}_{12} \mathrm{O}_{3} \cdot \mathrm{C}_{10} \mathrm{H}_{8} \mathrm{~N}_{2}$, (II) and $2 \mathrm{C}_{11} \mathrm{H}_{14} \mathrm{O}_{3} \cdot \mathrm{C}_{10} \mathrm{H}_{8} \mathrm{~N}_{2}$, (III), have been determined at $93 \mathrm{~K}$. Although the structure of (I) has been determined in the space group $P 2_{1}$ with $Z=4$ [Lai et al. (2008). J. Struct. Chem. 49, 1137-1140], the present study shows that the space group is $P 2_{1} / n$ with $Z=4$. In each crystal, the components are linked by $\mathrm{O}-$ $\mathrm{H} \cdots \mathrm{N}$ hydrogen bonds, forming a linear hydrogen-bonded 2:1 unit of the acid and the base. The 2:1 unit of (I) adopts nearly pseudo- $C_{2}$ symmetry, viz. twofold rotation around an axis passing through the mid-point of the central $\mathrm{C}-\mathrm{C}$ bond of 4,4'-bipyridyl, while the units of (II) and (III), except for the terminal alkyl chains, have pseudo-inversion symmetry. The 2:1 units of (I), (II) and (III) are linked via $\mathrm{C}-\mathrm{H} \cdots \mathrm{O}$ hydrogen bonds, forming sheet, double-tape and tape structures, respectively.

\section{Chemical context}

The 4-alkoxybenzoic acid-4,4'-bipyridyl (2/1) system, in which the two acids and the base are held together by intermolecular $\mathrm{O}-\mathrm{H} \cdots \mathrm{N}$ hydrogen bonds, shows thermotropic liquid crystallinity (Kato et al., 1990, 1993; Grunert et al., 1997). The compounds of 4-methoxy-, 4-ethoxy- and 4-n-propoxybenzoic acid show nematic phases, while the compound of 4- $n$-butoxybenzoic acid exhibits a smectic A phase and then a nematic phase with increasing temperature (Kato et al., 1990, 1993). The crystal structure of 4-methoxybenzoic acid-4,4'-bipyridyl (2/1) was reported recently (Mukherjee \& Desiraju, 2014; Ramon et al., 2014). Although the structure of 4-ethoxybenzoic acid-4,4'-bipyridyl (2/1) in space group $P 2_{1}$ was also reported (Lai et al., 2008), the molecular structure is distorted probably due to the wrong choice of space group. In the present study, we have analysed the structure of 4-ethoxybenzoic acid-4,4'-bipyridyl (2/1), (I), as well as the structures of 4- $n$-propoxybenzoic acid-4,4'-bipyridyl (2/1), (II), and 4- $n$ butoxybenzoic acid-4,4'-bipyridyl(2/1), (III).

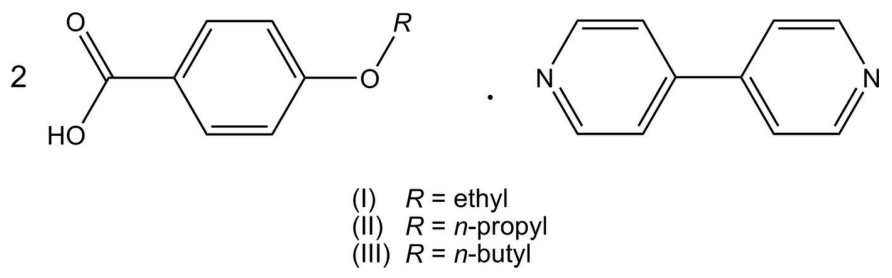




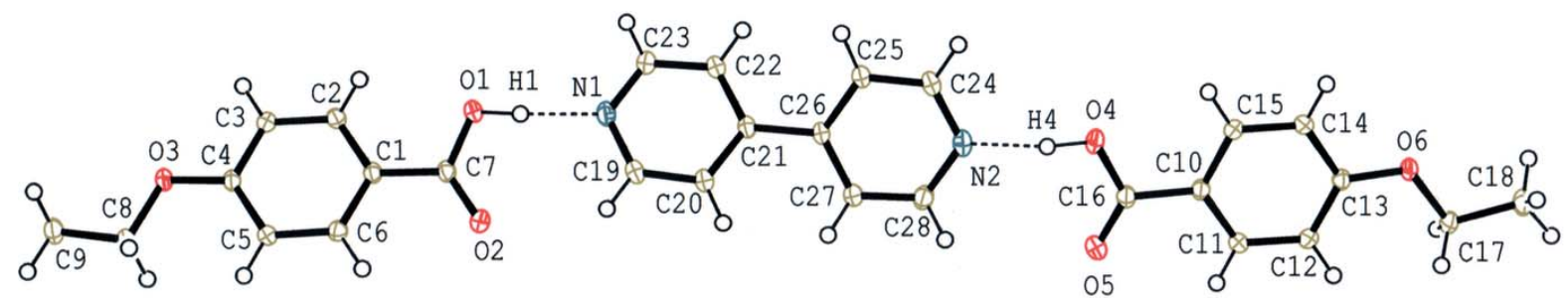

Figure 1

The molecular structure of compound (I), showing the atom-numbering scheme. Displacement ellipsoids of non- $\mathrm{H}$ atoms are drawn at the $50 \%$ probability level and $\mathrm{H}$ atoms are drawn as circles of arbitrary size. The $\mathrm{O}-\mathrm{H} \cdots \mathrm{N}$ hydrogen bonds are indicated by dashed lines.

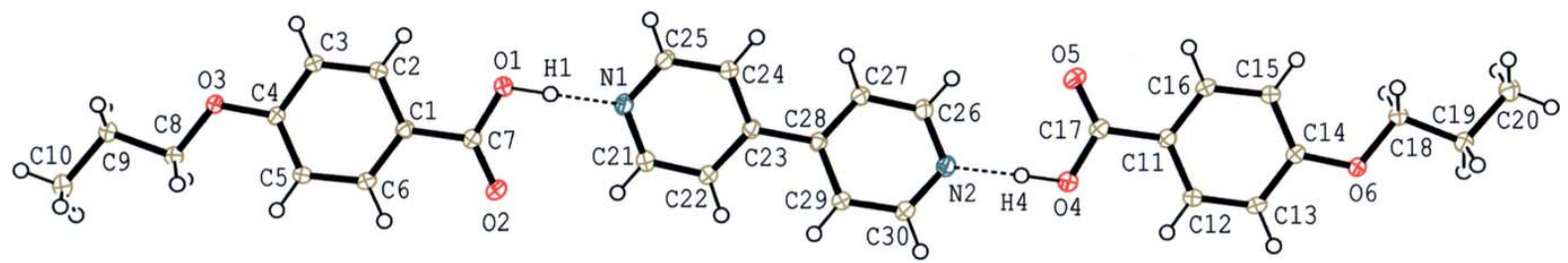

Figure 2

The molecular structure of compound (II), showing the atom-numbering scheme. Displacement ellipsoids of non- $\mathrm{H}$ atoms are drawn at the $50 \%$ probability level and $\mathrm{H}$ atoms are drawn as circles of arbitrary size. The $\mathrm{O}-\mathrm{H} \cdots \mathrm{N}$ hydrogen bonds are indicated by dashed lines.

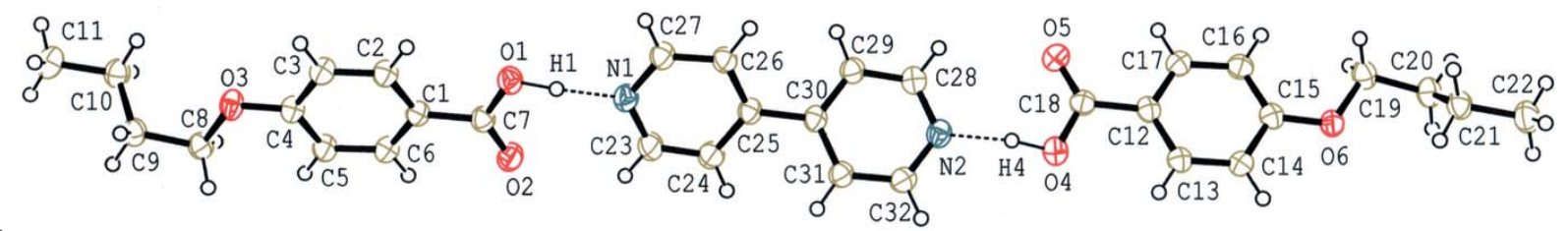

Figure 3

The molecular structure of compound (III), showing the atom-numbering scheme. Displacement ellipsoids of non-H atoms are drawn at the $50 \%$ probability level and $\mathrm{H}$ atoms are drawn as circles of arbitrary size. The $\mathrm{O}-\mathrm{H} \cdots \mathrm{N}$ hydrogen bonds are indicated by dashed lines.

\section{Structural commentary}

The molecular structure of (I) is shown in Fig. 1. Compound (I) crystallizes in the space group $P 2_{1} / n$ with $Z=4$. For the structure (space group $P 2_{1}$ ) previously determined by Lai et al. (2008), ADDSYM in PLATON (Spek, 2009) detected missed symmetry elements, viz. a centre of inversion and a glide plane. The molecular structures of (II) and (III) are shown in Figs. 2 and 3, respectively. The asymmetric units each comprise two crystallographically independent 4-alkoxybenzoic acid molecules and one 4,4'-bipyridyl molecule, and the two acids and the base are held together by $\mathrm{O}-\mathrm{H} \cdots \mathrm{N}$ hydrogen bonds (Tables 1, 2 and 3), forming a linear hydrogen-bonded 2:1 aggregate. Similar to the reported structure of the $2: 1$ unit of 4-methoxybenzoic acid-4,4'-bipyridyl (2/1) (Mukherjee \& Desiraju, 2014; Ramon et al., 2014), the 2:1 unit of (I) also adopts nearly pseudo- $C_{2}$ symmetry, viz. twofold rotation around an axis passing through the mid-point of the central C21-C26 bond of the 4,4'-bipyridyl molecule. On the other hand, the 2:1 units of (II) and (III), except for the terminal alkyl chains, have pseudo-inversion symmetry.

The dihedral angles between the pyridine rings of $4,4^{\prime}$ bipyridyl are 27.95 (5), 28.84 (4) and 38.76 (12) ${ }^{\circ}$ for (I), (II) and (III), respectively. The pyridine ring and the carboxyl group hydrogen-bonded to it are twisted slightly to each other. The dihedral angles between the N1/C19-C23 and O1/O2/C7 planes, and the $\mathrm{N} 2 / \mathrm{C} 24-\mathrm{C} 28$ and $\mathrm{O} 4 / \mathrm{O} 5 / \mathrm{C} 16$ planes are
$6.54(11)$ and $10.31(11)^{\circ}$, respectively, in (I), those between the N1/C21-C25 and O1/O2/C7 planes, and the N2/C26-C30 and $\mathrm{O} 4 / \mathrm{O} 5 / \mathrm{C} 17$ planes are $12.13(10)$ and $13.96(10)^{\circ}$, respectively, in (II), and those between the N1/C23-C27 and O1/O2/C7 planes, and the N2/C28-C32 and O4/O5/C18 planes are 13.7 (3) and $8.5(3)^{\circ}$, respectively, in (III).

The molecular structures of the ethoxy- and propoxybenzoic acids in (I) and (II) are approximately planar. The dihedral angles made by the benzene ring with the carboxyl group and the alkoxy group in each ethoxybenzoic acid in (I) are $9.60(10), 1.13(11), 4.48(9)$ and $7.57(9)^{\circ}$, respectively, between the $\mathrm{C} 1-\mathrm{C} 6$ and $\mathrm{O} 1 / \mathrm{O} 2 / \mathrm{C} 7$ planes, the $\mathrm{C} 10-\mathrm{C} 15$ and $\mathrm{O} 4 / \mathrm{O} 5 / \mathrm{C} 16$ planes, the $\mathrm{C} 1-\mathrm{C} 6$ and $\mathrm{O} 3 / \mathrm{C} 8 / \mathrm{C} 9$ planes, and the $\mathrm{C} 10-\mathrm{C} 15$ and $\mathrm{O} 6 / \mathrm{C} 17 / \mathrm{C} 18$ planes. The corresponding dihedral angles in (II) are $2.42(10), 2.48(10), 2.96(7)$ and $5.82(7)^{\circ}$, respectively, between the $\mathrm{C} 1-\mathrm{C} 6$ and $\mathrm{O} 1 / \mathrm{O} 2 / \mathrm{C} 7$ planes, the $\mathrm{C} 11-\mathrm{C} 16$ and $\mathrm{O} 4 / \mathrm{O} 5 / \mathrm{C} 17$ planes, the $\mathrm{C} 1-\mathrm{C} 6$ and $\mathrm{O} 3 / \mathrm{C} 8 / \mathrm{C} 9 /$ C10 planes, and the C11-C16 and O6/C18/C19/C20 planes. The butoxybenzoic acid molecules in (III) are also planar, except for the terminal ethyl groups which deviate from the molecular plane with dihedral angles of $66.6(3)$ and $60.7(3)^{\circ}$, respectively, between the $\mathrm{C} 4 / \mathrm{O} 3 / \mathrm{C} 8$ and $\mathrm{C} 9 / \mathrm{C} 10 / \mathrm{C} 11$ planes, and the $\mathrm{C} 15 / \mathrm{O} 6 / \mathrm{C} 19$ and $\mathrm{C} 20 / \mathrm{C} 21 / \mathrm{C} 22$ planes. The dihedral angles made by the benzene ring with the carboxyl group and the alkoxy group are 5.6(3), $5.4(3), 5.2(2)$ and $4.3(2)^{\circ}$, respectively, between the $\mathrm{C} 1-\mathrm{C} 6$ and $\mathrm{O} 1 / \mathrm{O} 2 / \mathrm{C} 7$ planes, the 


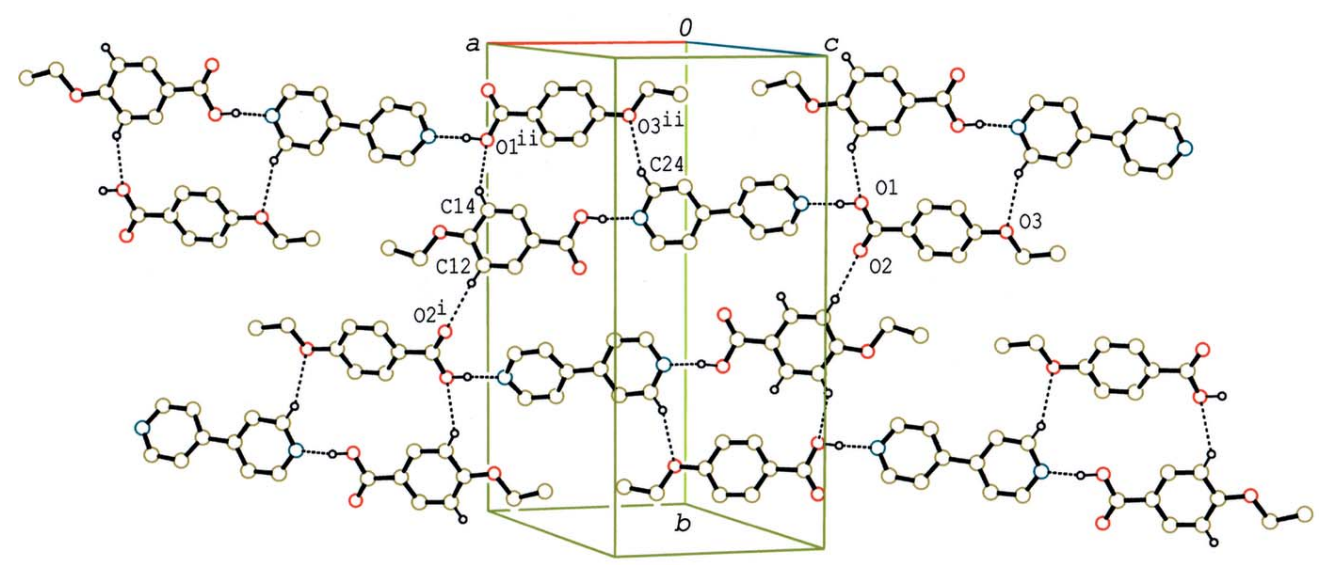

Figure 4

A partial packing diagram of compound (I), showing the sheet structure formed by $\mathrm{O}-\mathrm{H} \cdots \mathrm{N}$ and $\mathrm{C}-\mathrm{H} \cdots \mathrm{O}$ hydrogen bonds (dashed lines). $\mathrm{H}$ atoms not involved in the hydrogen bonds have been omitted. [Symmetry codes: (i) $-x+1,-y+1,-z+1$; (ii) $x+\frac{3}{2},-y+\frac{1}{2}, z-\frac{1}{2}$.]

Table 1

Hydrogen-bond geometry $\left(\AA{ }^{\circ}{ }^{\circ}\right)$ for (I).

$C g 1$ and $C g 2$ are the centroids of the $\mathrm{C} 1-\mathrm{C} 6$ and $\mathrm{C} 10-\mathrm{C} 15$ rings, respectively.

\begin{tabular}{lllll}
\hline$D-\mathrm{H} \cdots A$ & $D-\mathrm{H}$ & $\mathrm{H} \cdots A$ & $D \cdots A$ & $D-\mathrm{H} \cdots A$ \\
\hline $\mathrm{O} 1-\mathrm{H} 1 \cdots \mathrm{N} 1$ & $0.942(19)$ & $1.72(2)$ & $2.6587(11)$ & $177.2(19)$ \\
$\mathrm{O} 4-\mathrm{H} 4 \cdots \mathrm{N} 2$ & $0.948(19)$ & $1.690(19)$ & $2.6312(11)$ & $171.4(19)$ \\
$\mathrm{C} 12-\mathrm{H} 12 \cdots \mathrm{O} 2^{\mathrm{i}}$ & 0.95 & 2.43 & $3.3712(11)$ & 172 \\
$\mathrm{C} 14-\mathrm{H} 14 \cdots \mathrm{O} 1^{\mathrm{ii}}$ & 0.95 & 2.56 & $3.2288(11)$ & 128 \\
$\mathrm{C} 24-\mathrm{H} 24 \cdots 3^{\mathrm{ii}}$ & 0.95 & 2.57 & $3.4407(12)$ & 153 \\
$\mathrm{C} 9-\mathrm{H} 9 A \cdots C g 2^{\mathrm{ii}}$ & 0.98 & 2.68 & $3.6450(11)$ & 169 \\
$\mathrm{C} 18-\mathrm{H} 18 C \cdots C g 1^{\mathrm{iv}}$ & 0.98 & 2.67 & $3.6253(11)$ & 164
\end{tabular}

Symmetry codes: (i) $-x+1,-y+1,-z+1$; (ii) $x+\frac{3}{2},-y+\frac{1}{2}, z-\frac{1}{2}$; (iii) $x-2, y, z+1$; (iv) $x+2, y, z-1$.

Table 2

Hydrogen-bond geometry $\left(\AA,^{\circ}\right)$ for (II).

$C g 1$ and $C g 2$ are the centroids of the $\mathrm{C} 1-\mathrm{C} 6$ and $\mathrm{C} 11-\mathrm{C} 16$ rings, respectively.

\begin{tabular}{lllll}
\hline$D-\mathrm{H} \cdots A$ & $D-\mathrm{H}$ & $\mathrm{H} \cdots A$ & $D \cdots A$ & $D-\mathrm{H} \cdots A$ \\
\hline $\mathrm{O} 1-\mathrm{H} 1 \cdots \mathrm{N} 1$ & $1.03(2)$ & $1.61(2)$ & $2.6407(10)$ & $174.3(19)$ \\
$\mathrm{O} 4-\mathrm{H} 4 \cdots \mathrm{N} 2$ & $1.01(2)$ & $1.67(2)$ & $2.6728(11)$ & $173.9(18)$ \\
$\mathrm{C} 3-\mathrm{H} 3 \cdots 5^{\mathrm{i}}$ & 0.95 & 2.57 & $3.3981(11)$ & 146 \\
$\mathrm{C} 25-\mathrm{H} 25 \cdots \mathrm{O} 3^{\mathrm{ii}}$ & 0.95 & 2.57 & $3.4581(11)$ & 156 \\
$\mathrm{C} 9-\mathrm{H} 9 B \cdots C g 2^{\mathrm{iii}}$ & 0.99 & 2.84 & $3.6750(1)$ & 142 \\
$\mathrm{C} 19-\mathrm{H} 19 A \cdots C g 1^{\text {iv }}$ & 0.99 & 2.72 & $3.5781(1)$ & 146
\end{tabular}

Symmetry codes: (i) $-x+1,-y+2,-z$; (ii) $-x,-y+2,-z$; (iii) $x-2, y, z-1$; (iv) $x+2, y, z+1$.

Table 3

Hydrogen-bond geometry $\left(\AA,^{\circ}\right)$ for (III).

$C g 1$ and $C g 2$ are the centroids of the $\mathrm{C} 1-\mathrm{C} 6$ and $\mathrm{C} 12-\mathrm{C} 17$ rings, respectively.

\begin{tabular}{lllll}
\hline$D-\mathrm{H} \cdots A$ & $D-\mathrm{H}$ & $\mathrm{H} \cdots A$ & $D \cdots A$ & $D-\mathrm{H} \cdots A$ \\
\hline $\mathrm{O} 1-\mathrm{H} 1 \cdots \mathrm{N} 1$ & $1.04(3)$ & $1.56(3)$ & $2.600(3)$ & $173(3)$ \\
$\mathrm{O} 4-\mathrm{H} 4 \cdots \mathrm{N} 2$ & $1.00(4)$ & $1.64(4)$ & $2.636(3)$ & $172(4)$ \\
$\mathrm{C} 24-\mathrm{H} 24 \cdots \mathrm{O} 5^{\mathrm{i}}$ & 0.95 & 2.47 & $3.408(3)$ & 171 \\
$\mathrm{C} 29-\mathrm{H} 29 \cdots \mathrm{O} 2^{\mathrm{ii}}$ & 0.95 & 2.53 & $3.456(3)$ & 164 \\
$\mathrm{C} 2-\mathrm{H} 2 \cdots C g 2^{\mathrm{iii}}$ & 0.95 & 2.98 & $3.754(3)$ & 139 \\
$\mathrm{C} 8-\mathrm{H} 8 B \cdots C g 2^{\mathrm{iv}}$ & 0.99 & 2.68 & $3.518(3)$ & 143 \\
$\mathrm{C} 19-\mathrm{H} 19 B \cdots C g 1^{\mathrm{v}}$ & 0.99 & 2.77 & $3.586(3)$ & 140 \\
\hline
\end{tabular}

Symmetry codes: (i) $x, y-1, z$; (ii) $x, y+1, z$; (iii) $-x,-y+1,-z$; (iv) $-x+1,-y,-z$; (v) $-x+1,-y+1,-z$.
$\mathrm{C} 12-\mathrm{C} 17$ and $\mathrm{O} 4 / \mathrm{O} 5 / \mathrm{C} 18$ planes, the $\mathrm{C} 1-\mathrm{C} 6$ and $\mathrm{O} 3 / \mathrm{C} 8 / \mathrm{C} 9$ planes, and the C11-C16 and O6/C19/C20 planes.

\section{Supramolecular features}

In the crystal of (I), the 2:1 units are linked by $\mathrm{C}-\mathrm{H} \cdots \mathrm{O}$ hydrogen bonds (Table 1), forming a sheet structure parallel to (103) (Fig. 4). In addition, the units are stacked in a column through $\pi-\pi$ interactions between the acid and base rings along the $a$ axis (Fig. 5). The centroid-centroid distances between the $\mathrm{C} 1-\mathrm{C} 6$ and $\mathrm{N} 1 / \mathrm{C} 19-\mathrm{C} 23(x-1, y, z)$ rings, and between the $\mathrm{C} 10-\mathrm{C} 15$ and $\mathrm{N} 2 / \mathrm{C} 24-\mathrm{C} 28(x+1, y, z)$ rings are

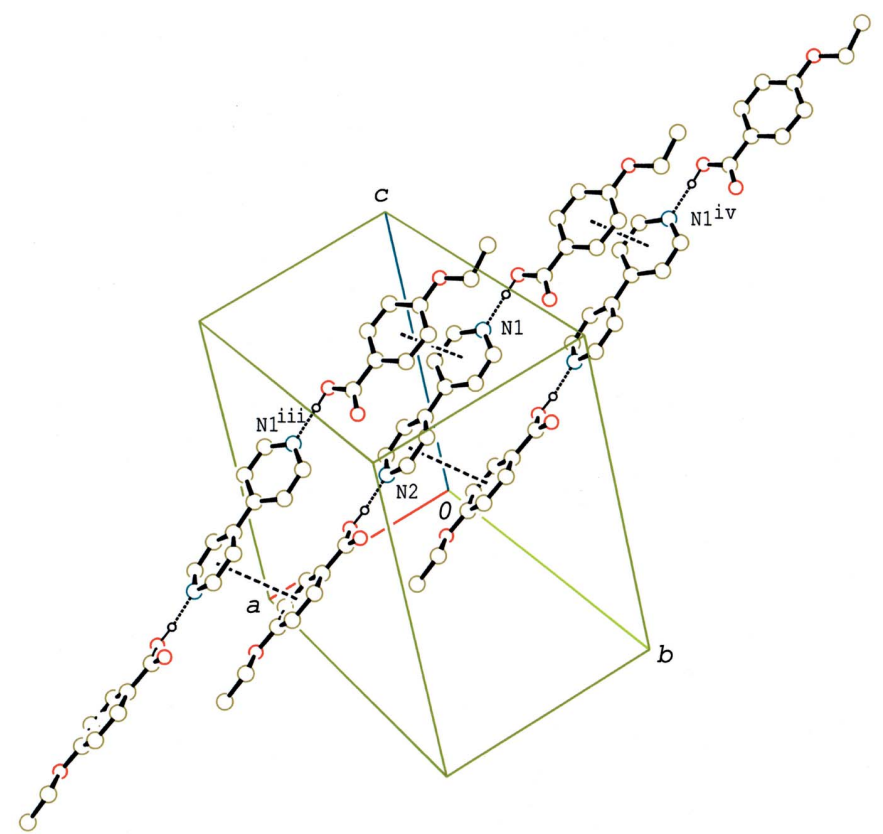

Figure 5

A partial packing diagram of compound (I), showing the column structure formed by $\pi-\pi$ stacking interactions (dashed lines). H atoms not involved in the $\mathrm{O}-\mathrm{H} \cdots \mathrm{N}$ hydrogen bonds have been omitted. [Symmetry codes: (iii) $x+1, y, z$; (iv) $x-1, y, z$.] 


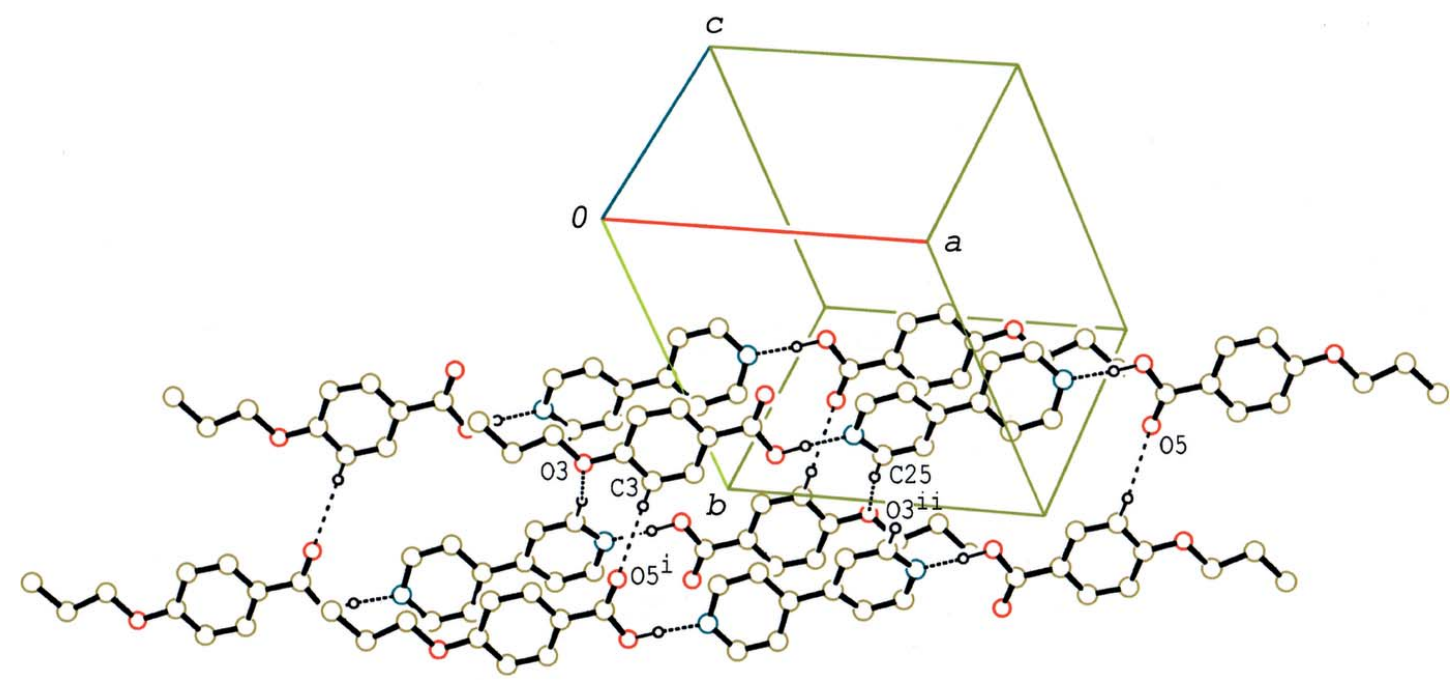

Figure 6

A partial packing diagram of compound (II), showing the double-tape structure formed by $\mathrm{C}-\mathrm{H} \cdots \mathrm{O}$ interactions. $\mathrm{H}$ atoms not involved in the $\mathrm{C}-$ $\mathrm{H} \cdots \mathrm{O}$ and $\mathrm{O}-\mathrm{H} \cdots \mathrm{N}$ hydrogen bonds (dashed lines) have been omitted. [Symmetry codes: (i) $-x+1,-y+2,-z ;$ (ii) $-x,-y+2,-z$.]

Figure 7

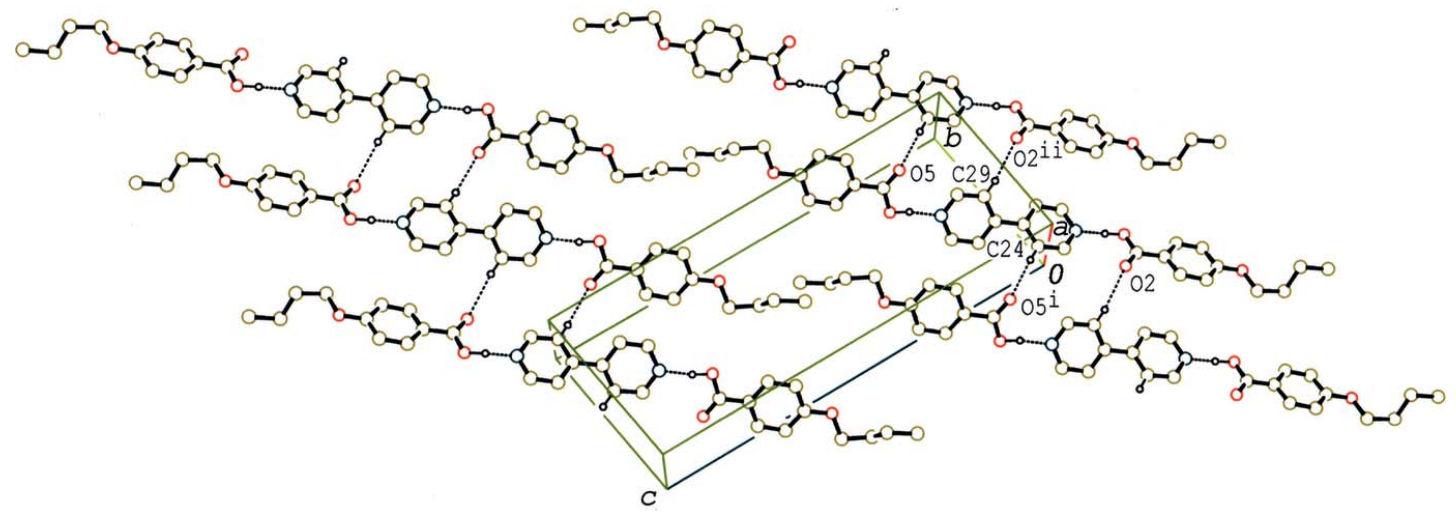

A partial packing diagram of compound (III), showing the tape structure formed by $\mathrm{C}-\mathrm{H} \cdots \mathrm{O}$ interactions. $\mathrm{H}$ atoms not involved in the $\mathrm{C}-\mathrm{H} \cdots \mathrm{O}$ and $\mathrm{O}-\mathrm{H} \cdots \mathrm{N}$ hydrogen bonds (dashed lines) have been omitted. [Symmetry codes: (i) $x, y-1, z$; (ii) $x, y+1, z$.]

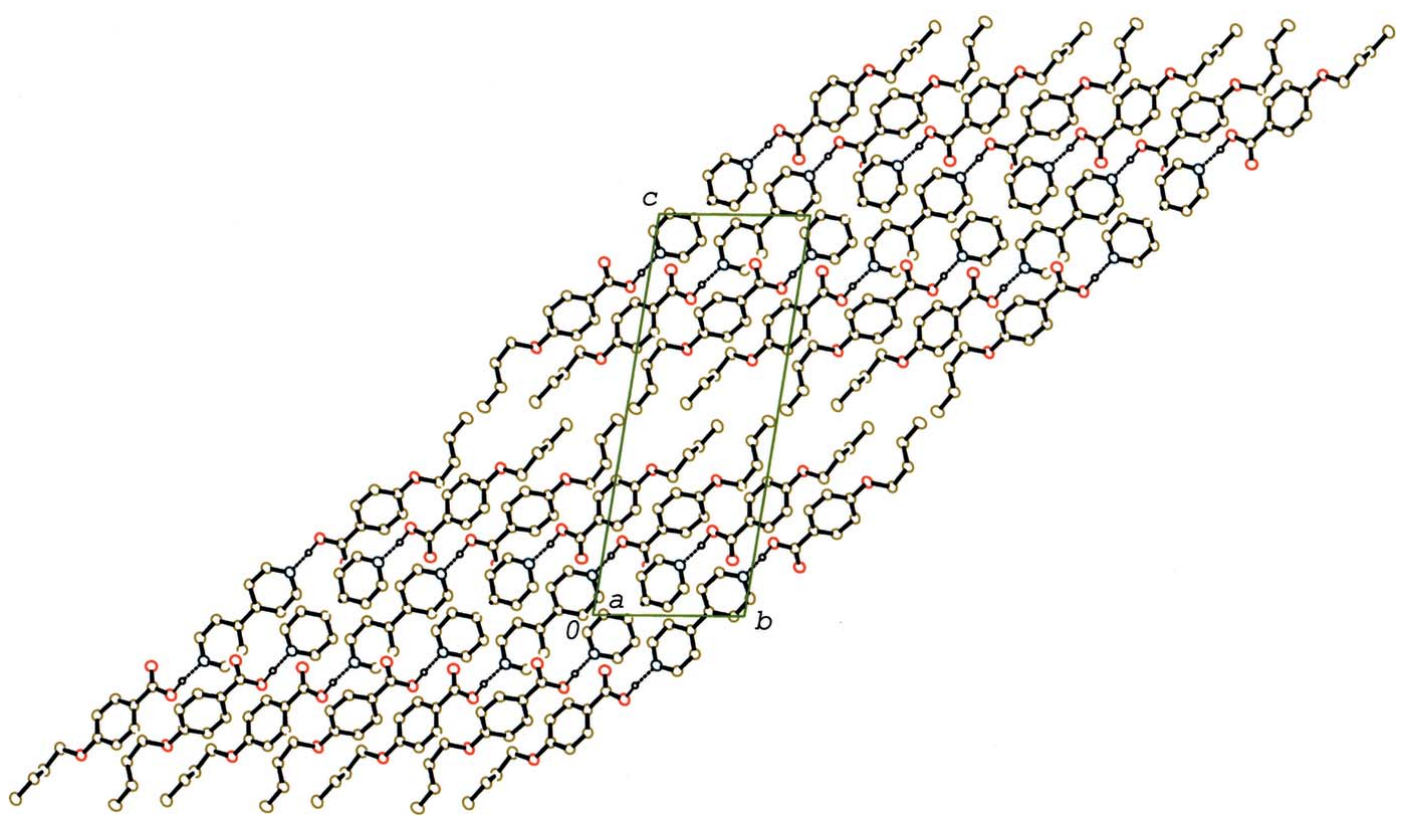

Figure 8

A packing diagram of compound (III) viewed along the $a$ axis, showing a layer aggregate. $\mathrm{H}$ atoms not involved in the $\mathrm{O}-\mathrm{H} \cdots \mathrm{N}$ hydrogen bonds (dashed lines) have been omitted. 
Table 4

Experimental details.

(I) (II)

Crystal data

Chemical formula

$M_{\mathrm{r}}$

Crystal system, space group

Temperature (K)

$a, b, c(\AA)$

$\alpha, \beta, \gamma\left(^{\circ}\right)$

$V\left(\AA^{3}\right)$

$Z$

Radiation type

$\mu\left(\mathrm{mm}^{-1}\right)$

Crystal size (mm)

Data collection

Diffractometer

No. of measured, independent and

observed $[I>2 \sigma(I)]$ reflections

$R_{\text {int }}$

$(\sin \theta / \lambda)_{\max }\left(\AA^{-1}\right)$

$2 \mathrm{C}_{9} \mathrm{H}_{10} \mathrm{O}_{3} \cdot \mathrm{C}_{10} \mathrm{H}_{8} \mathrm{~N}_{2}$

488.52

Monoclinic, $P 2_{1} / n$

93

9.1090 (2), 20.9348 (5), 12.8738 (4)

90, 102.9429 (10), 90

$2392.60(11)$

4

Mo $K \alpha$

0.10

$0.28 \times 0.25 \times 0.10$

Rigaku R-AXIS RAPIDII

28629, 6941, 6004

0.035

0.703

Refinement

$R\left[F^{2}>2 \sigma\left(F^{2}\right)\right], w R\left(F^{2}\right), S$

No. of reflections

No. of parameters

$\mathrm{H}$-atom treatment

$\Delta \rho_{\max }, \Delta \rho_{\min }\left(\mathrm{e} \AA^{-3}\right)$
(III)

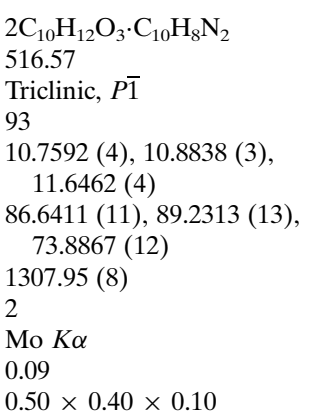

$2 \mathrm{C}_{11} \mathrm{H}_{14} \mathrm{O}_{3} \cdot \mathrm{C}_{10} \mathrm{H}_{8} \mathrm{~N}_{2}$

544.63

Triclinic, $P \overline{1}$

93

7.6645 (10), 8.5087 (13), 22.606 (3)

80.498 (3), 86.486 (3), 80.082 (3)

$1431.5(4)$

2

Mo $K \alpha$

0.09

$0.53 \times 0.41 \times 0.11$

Rigaku R-AXIS RAPIDII

$15909,7507,5980$

Rigaku R-AXIS RAPIDIIr $12433,5612,3432$

$\begin{array}{ll}0.069 & 0.075 \\ 0.703 & 0.617\end{array}$

$0.703-0.617$

$\begin{array}{ll}0.047,0.135,1.04 & 0.069,0.193,1.01 \\ 7507 & 5610 \\ 354 & 371 \\ \begin{array}{l}\mathrm{H} \text { atoms treated by a mixture of } \\ \text { independent and constrained }\end{array} & \begin{array}{c}\mathrm{H} \text { atoms treated by a mixture of } \\ \text { refinement }\end{array} \\ 0.35,-0.32 & \text { refinement } \\ & 0.24,-0.41\end{array}$

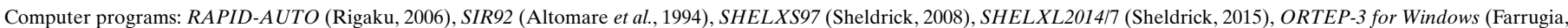
2012), CrystalStructure (Rigaku, 2010) and PLATON (Spek, 2009).

$3.7052(5)$ and $3.7752(6) \AA$, respectively. $\mathrm{C}-\mathrm{H} \cdots \pi$ interactions (Table 1) are also observed between the columns and between the sheets.

In the crystal of (II) and (III), the 2:1 units are linked by $\mathrm{C}-\mathrm{H} \cdots \mathrm{O}$ interactions (Tables 2 and 3), forming a doubletape structure along the $a$ axis (Fig. 6) and a tape structure along the $b$ axis (Fig. 7), respectively. Between the tapes in (II) and (III) $\mathrm{C}-\mathrm{H} \cdots \pi$ interactions are observed (Tables 2 and 3 ). A packing diagram of (III) viewed along the $a$ axis, which is approximately perpendicular to the mean plane of the 2:1 unit, is shown in Fig. 8. The units are arranged into a layer parallel to the $b c$ plane, which leads to a smectic structure. On the other hand, no such a layer structure is observed in compounds (I) and (II), which form nematic liquid phases.

\section{Database survey}

A search of the Cambridge Structural Database (Version 5.36, last update February 2015; Groom \& Allen, 2014) for cocrystals of 4,4'-bipyridyl with 4-alkoxybenzoic acid gave five structures (refcodes: NOPXIZ, ORASAC, RIRGUV, YAKVAI and YANCUM), except for 4-methoxybenzoic acid4,4'-bipyridyl (2/1) and 4-ethoxybenzoic acid-4,4'-bipyridyl $(2 / 1)$. Of these compounds, NOPXIZ, 4-[(S)-(-)-2-methylbutoxy]benzoic acid-4,4'-bipyridyl (2/10, shows smectic A and nematic phases (Grunert et al., 1997).

\section{Synthesis and crystallization}

Single crystals of compound (I) were obtained by slow evaporation from an acetone solution $(150 \mathrm{ml})$ of $4,4^{\prime}$-bipyridyl $(70 \mathrm{mg})$ with 4-ethoxybenzoic acid $(150 \mathrm{mg})$ at room temperature. Crystals of compounds (II) and (III) were obtained from ethanol solutions of 4,4'-bipyridyl with 4- $n$ propoxybenzoic acid and 4- $n$-butoxybenzoic acid, respectively, at room temperature [ethanol solution $(150 \mathrm{ml})$ of $4,4^{\prime}$-bipyridyl $(65 \mathrm{mg})$ and $4-n$-propoxybenzoic acid $(150 \mathrm{mg}$ ) for (II), and ethanol solution $(150 \mathrm{ml})$ of 4,4'-bipyridyl $(60 \mathrm{mg})$ and 4- $n$-butoxybenzoic acid (150 mg) for (III)].

Liquid crystalline phases of these compounds were confirmed by measurements of DSC (differential scanning calorimetry) and polarizing microscope. DSC measurements were performed by using Perkin Elmer Pyris 1 in the temperature range from $103 \mathrm{~K}$ to the melting temperature at a heating rate of $10 \mathrm{~K} \mathrm{~min}^{-1}$. Phase transition temperatures (K) and enthalpies $\left(\mathrm{kJ} \mathrm{mol}^{-1}\right)$ determined by DSC are as follows:

(I) 373 (2) $\left[5.4\right.$ (4)] $\mathrm{K}_{1} \rightarrow \mathrm{K}_{2}, 424$ (1) [50 (3)] $\mathrm{K}_{2} \rightarrow \mathrm{N}$, $442(1)[7.2(6)] \mathrm{N} \rightarrow \mathrm{I}$;

(II) 365 (1) $\left[2.9\right.$ (6)] $\mathrm{K}_{1} \rightarrow \mathrm{K}_{2}, 369$ (1) [3.9 (2)] $\mathrm{K}_{2} \rightarrow \mathrm{K}_{3}$, 417 (1) [39 (1)] $\mathrm{K}_{3} \rightarrow \mathrm{N}$, 430 (1) [5.7 (2)] N $\rightarrow$ I;

(III) 358 (1) $\left[2.5\right.$ (2)] $\mathrm{K}_{1} \rightarrow \mathrm{K}_{2}, 386$ (1) $\left[0.30\right.$ (3)] $\mathrm{K}_{2} \rightarrow \mathrm{K}_{3}$, 403 (1) [11.1 (5)] K $\mathrm{K}_{3} \rightarrow \mathrm{K}_{4}, 407$ (1) [24.5 (6)] $\mathrm{K}_{4} \rightarrow \mathrm{S}_{\mathrm{A}}, 425$ (1) [2.2(6)] $\mathrm{S}_{\mathrm{A}} \rightarrow \mathrm{N}, 432(1)[6.4(1)] \mathrm{N} \rightarrow \mathrm{I}$. 
$\mathrm{K}_{\mathrm{i}}, \mathrm{S}_{\mathrm{A}}, \mathrm{N}$ and I denote crystal, smectic A, nematic and isotropic phases, respectively. The observed transition temperatures and enthalpies from the solid phase to the liquid crystalline phase are in good agreement with those reported Kato et al. $(1990,1993)$. Some unreported thermal anomalies, 373 (2) K for (I), 365 (1) and 369 (1) K for (II), and 358 (1) and 386 (1) K for (III), were also observed.

\section{Refinement}

Crystal data, data collection and structure refinement details are summarized in Table 4. For all compounds, C-bound $\mathrm{H}$ atoms were positioned geometrically with $\mathrm{C}-\mathrm{H}=0.95-0.99 \AA$ and were refined as riding with $U_{\text {iso }}(\mathrm{H})=1.2 U_{\text {eq }}(\mathrm{C})$ or $1.5 U_{\text {eq }}$ (methyl C). The O-bound $\mathrm{H}$ atoms were located in a difference Fourier map and refined freely [refined $\mathrm{O}-\mathrm{H}=$ 0.942 (19)-1.04 (3) ̊]].

\section{References}

Altomare, A., Cascarano, G., Giacovazzo, C., Guagliardi, A., Burla, M. C., Polidori, G. \& Camalli, M. (1994). J. Appl. Cryst. 27, 435.

Farrugia, L. J. (2012). J. Appl. Cryst. 45, 849-854.

Groom, C. R. \& Allen, F. H. (2014). Angew. Chem. Int. Ed. 53, 662671.

Grunert, M., Howie, A., Kaeding, A. \& Imrie, C. T. (1997). J. Mater. Chem. 7, 211-214.

Kato, T., Fréchet, J. M. J., Wilson, P. G., Saito, T., Uryu, T., Fujishima, A., Jin, C. \& Kaneuchi, F. (1993). Chem. Mater. 5, 1094-1100.

Kato, T., Wilson, P. G., Fujishima, A. \& Fréchet, J. M. J. (1990). Chem. Lett. pp. 2003-2006.

Lai, L.-L., Lee, L.-J., Luo, D.-W., Liu, Y.-H. \& Wang, Y. (2008). J. Struct. Chem. 49, 1137-1140.

Mukherjee, A. \& Desiraju, G. R. (2014). Cryst. Growth Des. 14, 13751385.

Ramon, G., Davies, K. \& Nassimbeni, L. R. (2014). CrystEngComm, 16, 5802-5810.

Rigaku (2006). RAPID-AUTO. Rigaku Corporation, Tokyo, Japan. Rigaku (2010). CrystalStructure. Rigaku Corporation, Tokyo, Japan. Sheldrick, G. M. (2008). Acta Cryst. A64, 112-122.

Sheldrick, G. M. (2015). Acta Cryst. C71, 3-8.

Spek, A. L. (2009). Acta Cryst. D65, 148-155. 


\section{supporting information}

Acta Cryst. (2015). E71, 1290-1295 [https://doi.org/10.1107/S2056989015018435]

Crystal structures of three co-crystals of 4,4'-bipyridyl with 4-alkoxybenzoic acids: 4-ethoxybenzoic acid-4,4'-bipyridyl (2/1), 4-n-propoxybenzoic acid-4,4'bipyridyl (2/1) and 4-n-butoxybenzoic acid-4,4'-bipyridyl (2/1)

\section{Yohei Tabuchi, Kazuma Gotoh and Hiroyuki Ishida}

Computing details

For all compounds, data collection: RAPID-AUTO (Rigaku, 2006); cell refinement: RAPID-AUTO (Rigaku, 2006); data reduction: RAPID-AUTO (Rigaku, 2006). Program(s) used to solve structure: SIR92 (Altomare et al., 1994) for (I); SHELXS97 (Sheldrick, 2008) for (II), (III). For all compounds, program(s) used to refine structure: SHELXL2014/7 (Sheldrick, 2015); molecular graphics: ORTEP-3 for Windows (Farrugia, 2012); software used to prepare material for publication: CrystalStructure (Rigaku, 2010) and PLATON (Spek, 2009).

(I) 4-Ethoxybenzoic acid-4,4'-bipyridyl (2/1)

\section{Crystal data}

$2 \mathrm{C}_{9} \mathrm{H}_{10} \mathrm{O}_{3} \cdot \mathrm{C}_{10} \mathrm{H}_{8} \mathrm{~N}_{2}$

$M_{r}=488.52$

Monoclinic, $P 2{ }_{1} / n$

$a=9.1090(2) \AA$

$b=20.9348(5) \AA$

$c=12.8738(4) \AA$

$\beta=102.9429(10)^{\circ}$

$V=2392.60(11) \AA^{3}$

$Z=4$

\section{Data collection}

Rigaku R-AXIS RAPIDII diffractometer

Detector resolution: 10.000 pixels $\mathrm{mm}^{-1}$

$\omega$ scans

28629 measured reflections

6941 independent reflections

Refinement

Refinement on $F^{2}$

Least-squares matrix: full

$R\left[F^{2}>2 \sigma\left(F^{2}\right)\right]=0.045$

$w R\left(F^{2}\right)=0.127$

$S=1.04$

6941 reflections

335 parameters

0 restraints
$F(000)=1032.00$

$D_{\mathrm{x}}=1.356 \mathrm{Mg} \mathrm{m}^{-3}$

Mo $K \alpha$ radiation, $\lambda=0.71075 \AA$

Cell parameters from 23666 reflections

$\theta=3.0-30.0^{\circ}$

$\mu=0.10 \mathrm{~mm}^{-1}$

$T=93 \mathrm{~K}$

Needle, colorless

$0.28 \times 0.25 \times 0.10 \mathrm{~mm}$

6004 reflections with $I>2 \sigma(I)$

$R_{\text {int }}=0.035$

$\theta_{\max }=30.0^{\circ}$

$h=-12 \rightarrow 12$

$k=-28 \rightarrow 29$

$l=-18 \rightarrow 18$

Primary atom site location: structure-invariant direct methods

Secondary atom site location: difference Fourier map

Hydrogen site location: mixed

$\mathrm{H}$ atoms treated by a mixture of independent and constrained refinement 
$w=1 /\left[\sigma^{2}\left(F_{\mathrm{o}}^{2}\right)+(0.0817 P)^{2}+0.3767 P\right]$

where $P=\left(F_{\mathrm{o}}^{2}+2 F_{\mathrm{c}}^{2}\right) / 3$

$(\Delta / \sigma)_{\max }=0.001$

$$
\Delta \rho_{\max }=0.39 \text { e } \AA^{-3}
$$

\section{Special details}

Geometry. All esds (except the esd in the dihedral angle between two 1.s. planes) are estimated using the full covariance matrix. The cell esds are taken into account individually in the estimation of esds in distances, angles and torsion angles; correlations between esds in cell parameters are only used when they are defined by crystal symmetry. An approximate (isotropic) treatment of cell esds is used for estimating esds involving 1.s. planes.

Refinement. Reflections were merged by SHELXL according to the crystal class for the calculation of statistics and refinement.

Fractional atomic coordinates and isotropic or equivalent isotropic displacement parameters $\left(\AA^{2}\right)$

\begin{tabular}{|c|c|c|c|c|}
\hline & $x$ & $y$ & $z$ & $U_{\text {iso }} * / U_{\text {eq }}$ \\
\hline $\mathrm{O} 1$ & $-0.33398(8)$ & $0.30866(3)$ & $0.77777(6)$ & $0.02333(16)$ \\
\hline $\mathrm{O} 2$ & $-0.37344(8)$ & $0.40959(3)$ & $0.72097(6)$ & $0.02177(15)$ \\
\hline $\mathrm{O} 3$ & $-0.92113(7)$ & $0.36336(3)$ & $0.94659(6)$ & $0.01856(14)$ \\
\hline $\mathrm{O} 4$ & $0.74748(8)$ & $0.35124(3)$ & $0.34692(6)$ & $0.02249(16)$ \\
\hline $\mathrm{O} 5$ & $0.77838(8)$ & $0.45585(3)$ & $0.38227(6)$ & $0.02363(16)$ \\
\hline O6 & $1.33565(7)$ & $0.38813(3)$ & $0.17479(6)$ & $0.01967(15)$ \\
\hline N1 & $-0.10882(9)$ & $0.31350(4)$ & $0.67751(7)$ & $0.02016(17)$ \\
\hline N2 & $0.51947(9)$ & $0.35033(4)$ & $0.44136(7)$ & $0.02191(17)$ \\
\hline $\mathrm{C} 1$ & $-0.54350(10)$ & $0.36269(4)$ & $0.81521(7)$ & $0.01557(17)$ \\
\hline $\mathrm{C} 2$ & $-0.57223(10)$ & $0.31282(4)$ & $0.88043(7)$ & $0.01700(17)$ \\
\hline $\mathrm{H} 2$ & -0.5048 & 0.2777 & 0.8950 & $0.020^{*}$ \\
\hline $\mathrm{C} 3$ & $-0.69860(10)$ & $0.31438(4)$ & $0.92393(8)$ & $0.01764(17)$ \\
\hline H3 & -0.7169 & 0.2806 & 0.9686 & $0.021 *$ \\
\hline $\mathrm{C} 4$ & $-0.79903(10)$ & $0.36588(4)$ & $0.90180(7)$ & $0.01553(17)$ \\
\hline $\mathrm{C} 5$ & $-0.77115(10)$ & $0.41585(4)$ & $0.83675(7)$ & $0.01658(17)$ \\
\hline H5 & -0.8388 & 0.4509 & 0.8216 & $0.020^{*}$ \\
\hline C6 & $-0.64371(10)$ & $0.41374(4)$ & $0.79445(7)$ & $0.01672(17)$ \\
\hline H6 & -0.6246 & 0.4478 & 0.7505 & $0.020 *$ \\
\hline $\mathrm{C} 7$ & $-0.40997(10)$ & $0.36318(4)$ & $0.76677(7)$ & $0.01667(17)$ \\
\hline $\mathrm{C} 8$ & $-1.02432(10)$ & $0.41622(4)$ & $0.92520(8)$ & $0.01880(18)$ \\
\hline H8A & -1.0723 & 0.4179 & 0.8482 & $0.023 *$ \\
\hline H8B & -0.9696 & 0.4568 & 0.9453 & $0.023 *$ \\
\hline $\mathrm{C} 9$ & $-1.14267(11)$ & $0.40745(5)$ & $0.98921(8)$ & 0.02217 (19) \\
\hline H9A & -1.0945 & 0.4070 & 1.0653 & $0.033 *$ \\
\hline H9B & -1.1954 & 0.3669 & 0.9695 & $0.033 *$ \\
\hline $\mathrm{H} 9 \mathrm{C}$ & -1.2150 & 0.4427 & 0.9744 & $0.033 *$ \\
\hline $\mathrm{C} 10$ & $0.95579(10)$ & $0.40171(4)$ & $0.30216(7)$ & $0.01621(17)$ \\
\hline C11 & $1.04428(10)$ & $0.45571(4)$ & $0.30003(8)$ & $0.01774(17)$ \\
\hline H11 & 1.0160 & 0.4949 & 0.3273 & $0.021 *$ \\
\hline $\mathrm{C} 12$ & $1.17334(10)$ & $0.45373(4)$ & $0.25886(8)$ & $0.01782(18)$ \\
\hline H12 & 1.2328 & 0.4910 & 0.2585 & $0.021 *$ \\
\hline $\mathrm{C} 13$ & $1.21379(10)$ & $0.39607(4)$ & $0.21808(7)$ & 0.01639 (17) \\
\hline $\mathrm{C} 14$ & $1.12647(10)$ & $0.34139(4)$ & $0.22033(8)$ & $0.01837(18)$ \\
\hline H14 & 1.1544 & 0.3022 & 0.1928 & $0.022 *$ \\
\hline
\end{tabular}




$\begin{array}{lllll}\text { C15 } & 0.99956(10) & 0.34406(4) & 0.26245(8) & 0.01753(17) \\ \text { H15 } & 0.9416 & 0.3065 & 0.2645 & 0.021^{*} \\ \text { C16 } & 0.81942(10) & 0.40630(5) & 0.34748(7) & 0.01747(17) \\ \text { C17 } & 1.43238(10) & 0.44212(5) & 0.17318(8) & 0.02026(19) \\ \text { H17A } & 1.4830 & 0.4543 & 0.2468 & 0.024^{*} \\ \text { H17B } & 1.3729 & 0.4791 & 0.1388 & 0.024^{*} \\ \text { C18 } & 1.54764(11) & 0.42313(5) & 0.11099(8) & 0.0229(2) \\ \text { H18A } & 1.5998 & 0.3843 & 0.1421 & 0.034^{*} \\ \text { H18B } & 1.6209 & 0.4578 & 0.1139 & 0.034^{*} \\ \text { H18C } & 1.4970 & 0.4150 & 0.0366 & 0.034^{*} \\ \text { C19 } & -0.09136(10) & 0.36667(5) & 0.62319(8) & 0.01935(18) \\ \text { H19 } & -0.1662 & 0.3990 & 0.6159 & 0.023^{*} \\ \text { C20 } & 0.03056(10) & 0.37669(5) & 0.57728(7) & 0.01765(17) \\ \text { H20 } & 0.0388 & 0.4153 & 0.5402 & 0.021^{*} \\ \text { C21 } & 0.14130(10) & 0.32950(4) & 0.58603(7) & 0.01586(17) \\ \text { C22 } & 0.12277(10) & 0.27418(5) & 0.64248(8) & 0.01962(18) \\ \text { H22 } & 0.1954 & 0.2409 & 0.6508 & 0.024^{*} \\ \text { C23 } & -0.00263(11) & 0.26821(5) & 0.68641(8) & 0.02095(19) \\ \text { H23 } & -0.0137 & 0.2303 & 0.7245 & 0.025^{*} \\ \text { C24 } & 0.46423(11) & 0.29264(5) & 0.45714(9) & 0.0242(2) \\ \text { H24 } & 0.5105 & 0.2559 & 0.4352 & 0.029^{*} \\ \text { C25 } & 0.34301(11) & 0.28424(5) & 0.50393(9) & 0.0228(2) \\ \text { H25 } & 0.3080 & 0.2424 & 0.5140 & 0.027^{*} \\ \text { C26 } & 0.27224(10) & 0.33746(4) & 0.53636(7) & 0.01618(17) \\ \text { C27 } & 0.32923(10) & 0.39752(4) & 0.51958(8) & 0.01849(18) \\ \text { H27 } & 0.2847 & 0.4352 & 0.5400 & 0.022^{*} \\ \text { C28 } & 0.45223(11) & 0.40137(5) & 0.47250(8) & 0.02086(19) \\ \text { H28 } & 0.4906 & 0.4425 & 0.4619 & 0.025^{*} \\ \text { H1 } & -0.254(2) & 0.3118(9) & 0.7426(17) & 0.059(5)^{*} \\ \text { H4 } & 0.663(2) & 0.3549(10) & 0.3782(17) & 0.066(6)^{*}\end{array}$

Atomic displacement parameters $\left(\AA^{2}\right)$

\begin{tabular}{lllllll}
\hline & $U^{11}$ & $U^{22}$ & $U^{33}$ & $U^{12}$ & $U^{13}$ & $U^{23}$ \\
\hline O1 & $0.0216(3)$ & $0.0188(3)$ & $0.0345(4)$ & $0.0023(3)$ & $0.0167(3)$ & $0.0000(3)$ \\
O2 & $0.0200(3)$ & $0.0207(3)$ & $0.0274(4)$ & $-0.0013(3)$ & $0.0114(3)$ & $0.0013(3)$ \\
O3 & $0.0148(3)$ & $0.0197(3)$ & $0.0238(3)$ & $0.0017(2)$ & $0.0099(2)$ & $0.0022(2)$ \\
O4 & $0.0194(3)$ & $0.0226(3)$ & $0.0293(4)$ & $-0.0033(3)$ & $0.0136(3)$ & $-0.0007(3)$ \\
O5 & $0.0217(3)$ & $0.0227(3)$ & $0.0293(4)$ & $0.0038(3)$ & $0.0117(3)$ & $-0.0003(3)$ \\
O6 & $0.0165(3)$ & $0.0182(3)$ & $0.0276(4)$ & $-0.0019(2)$ & $0.0120(3)$ & $-0.0021(3)$ \\
N1 & $0.0170(3)$ & $0.0235(4)$ & $0.0220(4)$ & $-0.0013(3)$ & $0.0086(3)$ & $-0.0038(3)$ \\
N2 & $0.0168(3)$ & $0.0281(4)$ & $0.0228(4)$ & $0.0005(3)$ & $0.0088(3)$ & $0.0003(3)$ \\
C1 & $0.0141(4)$ & $0.0167(4)$ & $0.0167(4)$ & $-0.0013(3)$ & $0.0053(3)$ & $-0.0030(3)$ \\
C2 & $0.0152(4)$ & $0.0166(4)$ & $0.0198(4)$ & $0.0006(3)$ & $0.0052(3)$ & $-0.0012(3)$ \\
C3 & $0.0163(4)$ & $0.0169(4)$ & $0.0207(4)$ & $-0.0003(3)$ & $0.0062(3)$ & $0.0019(3)$ \\
C4 & $0.0134(4)$ & $0.0176(4)$ & $0.0165(4)$ & $-0.0009(3)$ & $0.0054(3)$ & $-0.0019(3)$ \\
C5 & $0.0161(4)$ & $0.0157(4)$ & $0.0189(4)$ & $0.0009(3)$ & $0.0059(3)$ & $-0.0003(3)$ \\
C6 & $0.0173(4)$ & $0.0165(4)$ & $0.0175(4)$ & $-0.0010(3)$ & $0.0061(3)$ & $-0.0007(3)$
\end{tabular}




$\begin{array}{lllllll}\text { C7 } & 0.0150(4) & 0.0179(4) & 0.0181(4) & -0.0015(3) & 0.0058(3) & -0.0043(3) \\ \text { C8 } & 0.0169(4) & 0.0185(4) & 0.0228(4) & 0.0022(3) & 0.0082(3) & -0.0003(3) \\ \text { C9 } & 0.0175(4) & 0.0280(5) & 0.0230(5) & 0.0027(4) & 0.0087(3) & 0.0021(4) \\ \text { C10 } & 0.0148(4) & 0.0179(4) & 0.0168(4) & 0.0010(3) & 0.0051(3) & 0.0023(3) \\ \text { C11 } & 0.0177(4) & 0.0159(4) & 0.0209(4) & 0.0019(3) & 0.0070(3) & 0.0005(3) \\ \text { C12 } & 0.0164(4) & 0.0156(4) & 0.0227(4) & -0.0010(3) & 0.0070(3) & 0.0012(3) \\ \text { C13 } & 0.0142(4) & 0.0176(4) & 0.0183(4) & 0.0008(3) & 0.0056(3) & 0.0018(3) \\ \text { C14 } & 0.0179(4) & 0.0150(4) & 0.0240(4) & 0.0009(3) & 0.0084(3) & -0.0001(3) \\ \text { C15 } & 0.0171(4) & 0.0159(4) & 0.0209(4) & -0.0009(3) & 0.0069(3) & 0.0016(3) \\ \text { C16 } & 0.0153(4) & 0.0207(4) & 0.0172(4) & 0.0015(3) & 0.0053(3) & 0.0026(3) \\ \text { C17 } & 0.0181(4) & 0.0192(4) & 0.0260(5) & -0.0034(3) & 0.0104(4) & -0.0015(3) \\ \text { C18 } & 0.0187(4) & 0.0272(5) & 0.0255(5) & -0.0047(4) & 0.0108(4) & -0.0055(4) \\ \text { C19 } & 0.0159(4) & 0.0230(4) & 0.0201(4) & 0.0026(3) & 0.0061(3) & -0.0022(3) \\ \text { C20 } & 0.0159(4) & 0.0205(4) & 0.0174(4) & 0.0009(3) & 0.0057(3) & -0.0007(3) \\ \text { C21 } & 0.0134(4) & 0.0196(4) & 0.0153(4) & -0.0008(3) & 0.0047(3) & -0.0034(3) \\ \text { C22 } & 0.0173(4) & 0.0200(4) & 0.0233(4) & 0.0022(3) & 0.0084(3) & 0.0000(3) \\ \text { C23 } & 0.0204(4) & 0.0212(4) & 0.0237(4) & -0.0006(3) & 0.0102(4) & 0.0007(3) \\ \text { C24 } & 0.0212(4) & 0.0241(4) & 0.0313(5) & 0.0035(4) & 0.0141(4) & -0.0014(4) \\ \text { C25 } & 0.0218(4) & 0.0194(4) & 0.0313(5) & 0.0000(4) & 0.0147(4) & -0.0019(4) \\ \text { C26 } & 0.0139(4) & 0.0197(4) & 0.0160(4) & 0.0009(3) & 0.0055(3) & -0.0010(3) \\ \text { C27 } & 0.0186(4) & 0.0194(4) & 0.0191(4) & 0.0006(3) & 0.0077(3) & -0.0007(3) \\ \text { C28 } & 0.0201(4) & 0.0227(4) & 0.0218(4) & -0.0028(3) & 0.0090(3) & 0.0000(3)\end{array}$

Geometric parameters ( $\left.\AA,{ }^{\circ}\right)$

\begin{tabular}{|c|c|c|c|}
\hline $\mathrm{O} 1-\mathrm{C} 7$ & $1.3259(11)$ & $\mathrm{C} 10-\mathrm{C} 16$ & $1.4891(12)$ \\
\hline $\mathrm{O} 1-\mathrm{H} 1$ & $0.942(19)$ & $\mathrm{C} 11-\mathrm{C} 12$ & $1.3941(12)$ \\
\hline $\mathrm{O} 2-\mathrm{C} 7$ & $1.2208(12)$ & $\mathrm{C} 11-\mathrm{H} 11$ & 0.9500 \\
\hline $\mathrm{O} 3-\mathrm{C} 4$ & $1.3637(10)$ & $\mathrm{C} 12-\mathrm{C} 13$ & $1.3984(13)$ \\
\hline $\mathrm{O} 3-\mathrm{C} 8$ & $1.4380(11)$ & $\mathrm{C} 12-\mathrm{H} 12$ & 0.9500 \\
\hline $\mathrm{O} 4-\mathrm{C} 16$ & $1.3252(12)$ & $\mathrm{C} 13-\mathrm{C} 14$ & $1.3979(12)$ \\
\hline $\mathrm{O} 4-\mathrm{H} 4$ & 0.948 (19) & $\mathrm{C} 14-\mathrm{C} 15$ & $1.3836(12)$ \\
\hline $\mathrm{O} 5-\mathrm{C} 16$ & $1.2213(12)$ & $\mathrm{C} 14-\mathrm{H} 14$ & 0.9500 \\
\hline $\mathrm{O} 6-\mathrm{C} 13$ & $1.3592(10)$ & $\mathrm{C} 15-\mathrm{H} 15$ & 0.9500 \\
\hline $\mathrm{O} 6-\mathrm{C} 17$ & $1.4361(11)$ & $\mathrm{C} 17-\mathrm{C} 18$ & $1.5092(13)$ \\
\hline $\mathrm{N} 1-\mathrm{C} 23$ & 1.3409 (13) & C17-H17A & 0.9900 \\
\hline $\mathrm{N} 1-\mathrm{C} 19$ & $1.3429(13)$ & C17-H17B & 0.9900 \\
\hline $\mathrm{N} 2-\mathrm{C} 28$ & $1.3370(13)$ & $\mathrm{C} 18-\mathrm{H} 18 \mathrm{~A}$ & 0.9800 \\
\hline $\mathrm{N} 2-\mathrm{C} 24$ & $1.3412(14)$ & C18-H18B & 0.9800 \\
\hline $\mathrm{C} 1-\mathrm{C} 6$ & $1.3920(12)$ & $\mathrm{C} 18-\mathrm{H} 18 \mathrm{C}$ & 0.9800 \\
\hline $\mathrm{C} 1-\mathrm{C} 2$ & $1.4006(13)$ & $\mathrm{C} 19-\mathrm{C} 20$ & $1.3859(12)$ \\
\hline $\mathrm{C} 1-\mathrm{C} 7$ & $1.4860(12)$ & C19-H19 & 0.9500 \\
\hline $\mathrm{C} 2-\mathrm{C} 3$ & $1.3887(12)$ & $\mathrm{C} 20-\mathrm{C} 21$ & $1.3983(12)$ \\
\hline $\mathrm{C} 2-\mathrm{H} 2$ & 0.9500 & $\mathrm{C} 20-\mathrm{H} 20$ & 0.9500 \\
\hline $\mathrm{C} 3-\mathrm{C} 4$ & $1.4012(12)$ & $\mathrm{C} 21-\mathrm{C} 22$ & $1.3974(13)$ \\
\hline $\mathrm{C} 3-\mathrm{H} 3$ & 0.9500 & $\mathrm{C} 21-\mathrm{C} 26$ & $1.4835(12)$ \\
\hline $\mathrm{C} 4-\mathrm{C} 5$ & $1.3984(12)$ & $\mathrm{C} 22-\mathrm{C} 23$ & $1.3892(12)$ \\
\hline $\mathrm{C} 5-\mathrm{C} 6$ & $1.3890(12)$ & $\mathrm{C} 22-\mathrm{H} 22$ & 0.9500 \\
\hline
\end{tabular}




\begin{tabular}{|c|c|c|c|}
\hline $\mathrm{C} 5-\mathrm{H} 5$ & 0.9500 & $\mathrm{C} 23-\mathrm{H} 23$ & 0.9500 \\
\hline C6- 6 6 & 0.9500 & $\mathrm{C} 24-\mathrm{C} 25$ & $1.3825(13)$ \\
\hline $\mathrm{C} 8-\mathrm{C} 9$ & $1.5077(13)$ & $\mathrm{C} 24-\mathrm{H} 24$ & 0.9500 \\
\hline $\mathrm{C} 8-\mathrm{H} 8 \mathrm{~A}$ & 0.9900 & $\mathrm{C} 25-\mathrm{C} 26$ & $1.3971(13)$ \\
\hline $\mathrm{C} 8-\mathrm{H} 8 \mathrm{~B}$ & 0.9900 & $\mathrm{C} 25-\mathrm{H} 25$ & 0.9500 \\
\hline C9-H9A & 0.9800 & $\mathrm{C} 26-\mathrm{C} 27$ & $1.3956(13)$ \\
\hline C9-H9B & 0.9800 & $\mathrm{C} 27-\mathrm{C} 28$ & $1.3905(12)$ \\
\hline $\mathrm{C} 9-\mathrm{H} 9 \mathrm{C}$ & 0.9800 & $\mathrm{C} 27-\mathrm{H} 27$ & 0.9500 \\
\hline $\mathrm{C} 10-\mathrm{C} 11$ & $1.3923(12)$ & $\mathrm{C} 28-\mathrm{H} 28$ & 0.9500 \\
\hline $\mathrm{C} 10-\mathrm{C} 15$ & $1.4030(12)$ & & \\
\hline $\mathrm{C} 7-\mathrm{O} 1-\mathrm{H} 1$ & $109.2(12)$ & $\mathrm{C} 15-\mathrm{C} 14-\mathrm{C} 13$ & $120.28(8)$ \\
\hline $\mathrm{C} 4-\mathrm{O} 3-\mathrm{C} 8$ & $116.70(7)$ & $\mathrm{C} 15-\mathrm{C} 14-\mathrm{H} 14$ & 119.9 \\
\hline $\mathrm{C} 16-\mathrm{O} 4-\mathrm{H} 4$ & $112.0(13)$ & $\mathrm{C} 13-\mathrm{C} 14-\mathrm{H} 14$ & 119.9 \\
\hline $\mathrm{C} 13-\mathrm{O} 6-\mathrm{C} 17$ & $118.02(7)$ & $\mathrm{C} 14-\mathrm{C} 15-\mathrm{C} 10$ & $120.54(8)$ \\
\hline $\mathrm{C} 23-\mathrm{N} 1-\mathrm{C} 19$ & $117.60(8)$ & $\mathrm{C} 14-\mathrm{C} 15-\mathrm{H} 15$ & 119.7 \\
\hline $\mathrm{C} 28-\mathrm{N} 2-\mathrm{C} 24$ & $117.50(8)$ & $\mathrm{C} 10-\mathrm{C} 15-\mathrm{H} 15$ & 119.7 \\
\hline $\mathrm{C} 6-\mathrm{C} 1-\mathrm{C} 2$ & $119.00(8)$ & $\mathrm{O} 5-\mathrm{C} 16-\mathrm{O} 4$ & $123.31(8)$ \\
\hline $\mathrm{C} 6-\mathrm{C} 1-\mathrm{C} 7$ & $118.51(8)$ & $\mathrm{O} 5-\mathrm{C} 16-\mathrm{C} 10$ & $123.32(8)$ \\
\hline $\mathrm{C} 2-\mathrm{C} 1-\mathrm{C} 7$ & $122.49(8)$ & $\mathrm{O} 4-\mathrm{C} 16-\mathrm{C} 10$ & $113.37(8)$ \\
\hline $\mathrm{C} 3-\mathrm{C} 2-\mathrm{C} 1$ & $120.48(8)$ & $\mathrm{O} 6-\mathrm{C} 17-\mathrm{C} 18$ & $107.60(8)$ \\
\hline $\mathrm{C} 3-\mathrm{C} 2-\mathrm{H} 2$ & 119.8 & $\mathrm{O} 6-\mathrm{C} 17-\mathrm{H} 17 \mathrm{~A}$ & 110.2 \\
\hline $\mathrm{C} 1-\mathrm{C} 2-\mathrm{H} 2$ & 119.8 & $\mathrm{C} 18-\mathrm{C} 17-\mathrm{H} 17 \mathrm{~A}$ & 110.2 \\
\hline $\mathrm{C} 2-\mathrm{C} 3-\mathrm{C} 4$ & $119.90(8)$ & $\mathrm{O} 6-\mathrm{C} 17-\mathrm{H} 17 \mathrm{~B}$ & 110.2 \\
\hline $\mathrm{C} 2-\mathrm{C} 3-\mathrm{H} 3$ & 120.0 & $\mathrm{C} 18-\mathrm{C} 17-\mathrm{H} 17 \mathrm{~B}$ & 110.2 \\
\hline $\mathrm{C} 4-\mathrm{C} 3-\mathrm{H} 3$ & 120.0 & $\mathrm{H} 17 \mathrm{~A}-\mathrm{C} 17-\mathrm{H} 17 \mathrm{~B}$ & 108.5 \\
\hline $\mathrm{O} 3-\mathrm{C} 4-\mathrm{C} 5$ & $123.84(8)$ & $\mathrm{C} 17-\mathrm{C} 18-\mathrm{H} 18 \mathrm{~A}$ & 109.5 \\
\hline $\mathrm{O} 3-\mathrm{C} 4-\mathrm{C} 3$ & $116.20(8)$ & $\mathrm{C} 17-\mathrm{C} 18-\mathrm{H} 18 \mathrm{~B}$ & 109.5 \\
\hline $\mathrm{C} 5-\mathrm{C} 4-\mathrm{C} 3$ & $119.96(8)$ & $\mathrm{H} 18 \mathrm{~A}-\mathrm{C} 18-\mathrm{H} 18 \mathrm{~B}$ & 109.5 \\
\hline $\mathrm{C} 6-\mathrm{C} 5-\mathrm{C} 4$ & $119.40(8)$ & $\mathrm{C} 17-\mathrm{C} 18-\mathrm{H} 18 \mathrm{C}$ & 109.5 \\
\hline $\mathrm{C} 6-\mathrm{C} 5-\mathrm{H} 5$ & 120.3 & $\mathrm{H} 18 \mathrm{~A}-\mathrm{C} 18-\mathrm{H} 18 \mathrm{C}$ & 109.5 \\
\hline $\mathrm{C} 4-\mathrm{C} 5-\mathrm{H} 5$ & 120.3 & $\mathrm{H} 18 \mathrm{~B}-\mathrm{C} 18-\mathrm{H} 18 \mathrm{C}$ & 109.5 \\
\hline $\mathrm{C} 5-\mathrm{C} 6-\mathrm{C} 1$ & $121.25(8)$ & $\mathrm{N} 1-\mathrm{C} 19-\mathrm{C} 20$ & $123.18(8)$ \\
\hline $\mathrm{C} 5-\mathrm{C} 6-\mathrm{H} 6$ & 119.4 & $\mathrm{~N} 1-\mathrm{C} 19-\mathrm{H} 19$ & 118.4 \\
\hline $\mathrm{C} 1-\mathrm{C} 6-\mathrm{H} 6$ & 119.4 & $\mathrm{C} 20-\mathrm{C} 19-\mathrm{H} 19$ & 118.4 \\
\hline $\mathrm{O} 2-\mathrm{C} 7-\mathrm{O} 1$ & $123.00(8)$ & $\mathrm{C} 19-\mathrm{C} 20-\mathrm{C} 21$ & $119.42(9)$ \\
\hline $\mathrm{O} 2-\mathrm{C} 7-\mathrm{C} 1$ & $123.05(8)$ & $\mathrm{C} 19-\mathrm{C} 20-\mathrm{H} 20$ & 120.3 \\
\hline $\mathrm{O} 1-\mathrm{C} 7-\mathrm{C} 1$ & $113.95(8)$ & $\mathrm{C} 21-\mathrm{C} 20-\mathrm{H} 20$ & 120.3 \\
\hline $\mathrm{O} 3-\mathrm{C} 8-\mathrm{C} 9$ & $108.56(8)$ & $\mathrm{C} 22-\mathrm{C} 21-\mathrm{C} 20$ & $117.29(8)$ \\
\hline $\mathrm{O} 3-\mathrm{C} 8-\mathrm{H} 8 \mathrm{~A}$ & 110.0 & $\mathrm{C} 22-\mathrm{C} 21-\mathrm{C} 26$ & $121.30(8)$ \\
\hline $\mathrm{C} 9-\mathrm{C} 8-\mathrm{H} 8 \mathrm{~A}$ & 110.0 & $\mathrm{C} 20-\mathrm{C} 21-\mathrm{C} 26$ & $121.41(8)$ \\
\hline $\mathrm{O} 3-\mathrm{C} 8-\mathrm{H} 8 \mathrm{~B}$ & 110.0 & $\mathrm{C} 23-\mathrm{C} 22-\mathrm{C} 21$ & $119.50(8)$ \\
\hline $\mathrm{C} 9-\mathrm{C} 8-\mathrm{H} 8 \mathrm{~B}$ & 110.0 & $\mathrm{C} 23-\mathrm{C} 22-\mathrm{H} 22$ & 120.2 \\
\hline $\mathrm{H} 8 \mathrm{~A}-\mathrm{C} 8-\mathrm{H} 8 \mathrm{~B}$ & 108.4 & $\mathrm{C} 21-\mathrm{C} 22-\mathrm{H} 22$ & 120.2 \\
\hline $\mathrm{C} 8-\mathrm{C} 9-\mathrm{H} 9 \mathrm{~A}$ & 109.5 & $\mathrm{~N} 1-\mathrm{C} 23-\mathrm{C} 22$ & $123.01(9)$ \\
\hline $\mathrm{C} 8-\mathrm{C} 9-\mathrm{H} 9 \mathrm{~B}$ & 109.5 & $\mathrm{~N} 1-\mathrm{C} 23-\mathrm{H} 23$ & 118.5 \\
\hline $\mathrm{H} 9 \mathrm{~A}-\mathrm{C} 9-\mathrm{H} 9 \mathrm{~B}$ & 109.5 & $\mathrm{C} 22-\mathrm{C} 23-\mathrm{H} 23$ & 118.5 \\
\hline $\mathrm{C} 8-\mathrm{C} 9-\mathrm{H} 9 \mathrm{C}$ & 109.5 & $\mathrm{~N} 2-\mathrm{C} 24-\mathrm{C} 25$ & $122.95(9)$ \\
\hline
\end{tabular}




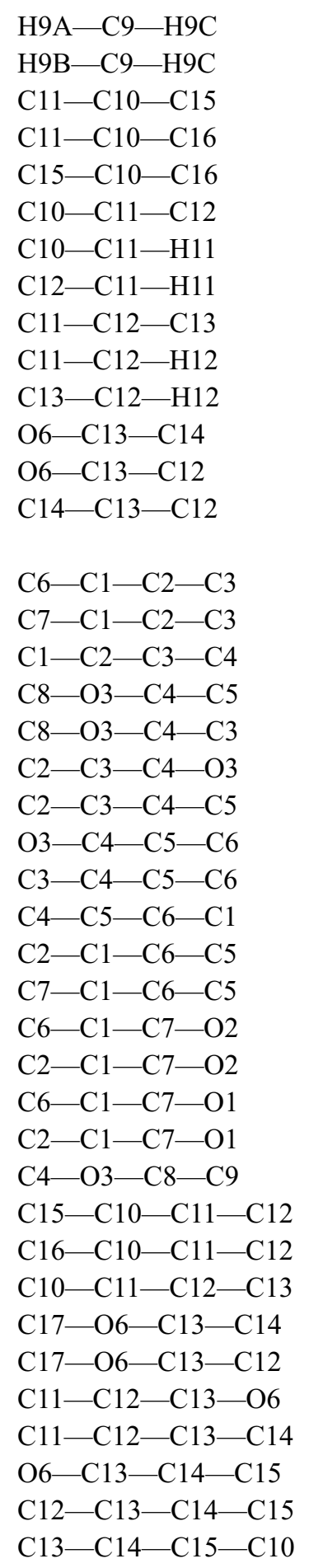

109.5

109.5

$118.58(8)$

$119.52(8)$

$121.89(8)$

$121.64(8)$

119.2

119.2

$118.94(8)$

120.5

120.5

$115.41(8)$

$124.58(8)$

$120.01(8)$

$-0.16(13)$

$-179.95(8)$

$0.57(14)$

$-0.72(13)$

$179.48(8)$

$179.25(8)$

$-0.56(14)$

$-179.65(8)$

$0.14(14)$

$0.28(14)$

$-0.27(14)$

$179.53(8)$

$9.58(13)$

$-170.62(9)$

$-170.43(8)$

$9.37(13)$

$-174.93(8)$

$0.53(14)$

$179.70(8)$

$0.48(14)$

$-178.14(8)$

$1.77(13)$

$179.23(9)$

$-0.87(14)$

$-179.85(8)$

$0.24(14)$

$0.80(14)$
N2- $224-\mathrm{H} 24$

$\mathrm{C} 25-\mathrm{C} 24-\mathrm{H} 24$

$\mathrm{C} 24-\mathrm{C} 25-\mathrm{C} 26$

$\mathrm{C} 24-\mathrm{C} 25-\mathrm{H} 25$

$\mathrm{C} 26-\mathrm{C} 25-\mathrm{H} 25$

$\mathrm{C} 27-\mathrm{C} 26-\mathrm{C} 25$

$\mathrm{C} 27-\mathrm{C} 26-\mathrm{C} 21$

$\mathrm{C} 25-\mathrm{C} 26-\mathrm{C} 21$

$\mathrm{C} 28-\mathrm{C} 27-\mathrm{C} 26$

$\mathrm{C} 28-\mathrm{C} 27-\mathrm{H} 27$

$\mathrm{C} 26-\mathrm{C} 27-\mathrm{H} 27$

$\mathrm{N} 2-\mathrm{C} 28-\mathrm{C} 27$

$\mathrm{N} 2-\mathrm{C} 28-\mathrm{H} 28$

$\mathrm{C} 27-\mathrm{C} 28-\mathrm{H} 28$

$\mathrm{C} 11-\mathrm{C} 10-\mathrm{C} 15-\mathrm{C} 14$

$\mathrm{C} 16-\mathrm{C} 10-\mathrm{C} 15-\mathrm{C} 14$

$\mathrm{C} 11-\mathrm{C} 10-\mathrm{C} 16-\mathrm{O} 5$

$\mathrm{C} 15-\mathrm{C} 10-\mathrm{C} 16-\mathrm{O} 5$

$\mathrm{C} 11-\mathrm{C} 10-\mathrm{C} 16-\mathrm{O} 4$

$\mathrm{C} 15-\mathrm{C} 10-\mathrm{C} 16-\mathrm{O} 4$

$\mathrm{C} 13-\mathrm{O} 6-\mathrm{C} 17-\mathrm{C} 18$

$\mathrm{C} 23-\mathrm{N} 1-\mathrm{C} 19-\mathrm{C} 20$

$\mathrm{N} 1-\mathrm{C} 19-\mathrm{C} 20-\mathrm{C} 21$

$\mathrm{C} 19-\mathrm{C} 20-\mathrm{C} 21-\mathrm{C} 22$

$\mathrm{C} 19-\mathrm{C} 20-\mathrm{C} 21-\mathrm{C} 26$

$\mathrm{C} 20-\mathrm{C} 21-\mathrm{C} 22-\mathrm{C} 23$

$\mathrm{C} 26-\mathrm{C} 21-\mathrm{C} 22-\mathrm{C} 23$

$\mathrm{C} 19-\mathrm{N} 1-\mathrm{C} 23-\mathrm{C} 22$

$\mathrm{C} 21-\mathrm{C} 22-\mathrm{C} 23-\mathrm{N} 1$

$\mathrm{C} 28-\mathrm{N} 2-\mathrm{C} 24-\mathrm{C} 25$

$\mathrm{N} 2-\mathrm{C} 24-\mathrm{C} 25-\mathrm{C} 26$

$\mathrm{C} 24-\mathrm{C} 25-\mathrm{C} 26-\mathrm{C} 27$

$\mathrm{C} 24-\mathrm{C} 25-\mathrm{C} 26-\mathrm{C} 21$

$\mathrm{C} 22-\mathrm{C} 21-\mathrm{C} 26-\mathrm{C} 27$

$\mathrm{C} 20-\mathrm{C} 21-\mathrm{C} 26-\mathrm{C} 27$

$\mathrm{C} 22-\mathrm{C} 21-\mathrm{C} 26-\mathrm{C} 25$

$\mathrm{C} 20-\mathrm{C} 21-\mathrm{C} 26-\mathrm{C} 25$

$\mathrm{C} 25-\mathrm{C} 26-\mathrm{C} 27-\mathrm{C} 28$

$\mathrm{C} 21-\mathrm{C} 26-\mathrm{C} 27-\mathrm{C} 28$

$\mathrm{C} 24-\mathrm{N} 2-\mathrm{C} 28-\mathrm{C} 27$

$\mathrm{C} 26-\mathrm{C} 27-\mathrm{C} 28-\mathrm{N} 2$
118.5

118.5

$119.73(9)$

120.1

120.1

$117.37(8)$

$122.05(8)$

$120.57(8)$

$118.92(8)$

120.5

120.5

123.53 (9)

118.2

118.2

$-1.18(14)$

$179.68(8)$

$1.11(14)$

$-179.75(9)$

$-178.52(8)$

$0.62(13)$

$-173.89(8)$

$-0.45(14)$

0.69 (14)

$-0.57(13)$

$178.73(8)$

$0.28(14)$

$-179.03(9)$

$0.13(15)$

$-0.05(15)$

$-0.27(16)$

$0.41(17)$

$-0.13(15)$

$179.56(9)$

$-152.77(9)$

27.95 (13)

27.55 (13)

$-151.72(10)$

$-0.26(14)$

$-179.94(9)$

$-0.16(15)$

$0.43(15)$

Hydrogen-bond geometry $\left(A,{ }^{o}\right)$

$C g 1$ and $C g 2$ are the centroids of the $\mathrm{C} 1-\mathrm{C} 6$ and $\mathrm{C} 10-\mathrm{C} 15$ rings, respectively.

\begin{tabular}{lllll}
\hline$D-\mathrm{H} \cdots A$ & $D-\mathrm{H}$ & $\mathrm{H} \cdots A$ & $D \cdots A$ & $D-\mathrm{H} \cdots A$ \\
\hline $\mathrm{O} 1-\mathrm{H} 1 \cdots \mathrm{N} 1$ & $0.942(19)$ & $1.72(2)$ & $2.6587(11)$ & $177.2(19)$
\end{tabular}




$\begin{array}{lllll}\mathrm{O} 4-\mathrm{H} 4 \cdots \mathrm{N} 2 & 0.948(19) & 1.690(19) & 2.6312(11) & 171.4(19) \\ \mathrm{C} 12-\mathrm{H} 12 \cdots \mathrm{O} 2^{\mathrm{i}} & 0.95 & 2.43 & 3.3712(11) & 172 \\ \mathrm{C} 14-\mathrm{H} 14 \cdots \mathrm{O} 1^{\text {ii }} & 0.95 & 2.56 & 3.2288(11) & 128 \\ \mathrm{C} 24-\mathrm{H} 24 \cdots \mathrm{O} 3^{\text {ii }} & 0.95 & 2.57 & 3.4407(12) & 153 \\ \mathrm{C} 9-\mathrm{H} 9 A \cdots C g 2^{\text {iii }} & 0.98 & 2.68 & 3.6450(11) & 169 \\ \mathrm{C} 18-\mathrm{H} 18 C \cdots C g 1^{\text {iv }} & 0.98 & 2.67 & 3.6253(11) & 164\end{array}$

Symmetry codes: (i) $-x+1,-y+1,-z+1$; (ii) $x+3 / 2,-y+1 / 2, z-1 / 2$; (iii) $x-2, y, z+1$; (iv) $x+2, y, z-1$.

(II) 4-n-Propoxybenzoic acid-4,4'-bipyridyl (2/1)

Crystal data

$2 \mathrm{C}_{10} \mathrm{H}_{12} \mathrm{O}_{3} \cdot \mathrm{C}_{10} \mathrm{H}_{8} \mathrm{~N}_{2}$

$M_{r}=516.57$

Triclinic, $P \overline{1}$

$a=10.7592(4) \AA$

$b=10.8838(3) \AA$

$c=11.6462(4) \AA$

$\alpha=86.6411(11)^{\circ}$

$\beta=89.2313(13)^{\circ}$

$\gamma=73.8867(12)^{\circ}$

$V=1307.95(8) \AA^{3}$

Data collection

Rigaku R-AXIS RAPIDII diffractometer

Detector resolution: 10.000 pixels $\mathrm{mm}^{-1}$

$\omega$ scans

15909 measured reflections

7507 independent reflections

\section{Refinement}

Refinement on $F^{2}$

Least-squares matrix: full

$R\left[F^{2}>2 \sigma\left(F^{2}\right)\right]=0.047$

$w R\left(F^{2}\right)=0.135$

$S=1.04$

7507 reflections

354 parameters

0 restraints

Primary atom site location: structure-invariant direct methods

Secondary atom site location: difference Fourier map

\section{Special details}

Geometry. All esds (except the esd in the dihedral angle between two 1.s. planes) are estimated using the full covariance matrix. The cell esds are taken into account individually in the estimation of esds in distances, angles and torsion angles; correlations between esds in cell parameters are only used when they are defined by crystal symmetry. An approximate (isotropic) treatment of cell esds is used for estimating esds involving l.s. planes.

Refinement. Reflections were merged by SHELXL according to the crystal class for the calculation of statistics and refinement.
$Z=2$

$F(000)=548.00$

$D_{\mathrm{x}}=1.312 \mathrm{Mg} \mathrm{m}^{-3}$

Mo $K \alpha$ radiation, $\lambda=0.71075 \AA$

Cell parameters from 13551 reflections

$\theta=3.0-30.0^{\circ}$

$\mu=0.09 \mathrm{~mm}^{-1}$

$T=93 \mathrm{~K}$

Block, colorless

$0.50 \times 0.40 \times 0.10 \mathrm{~mm}$

5980 reflections with $I>2 \sigma(I)$

$R_{\text {int }}=0.069$

$\theta_{\text {max }}=30.0^{\circ}, \theta_{\text {min }}=3.0^{\circ}$

$h=-15 \rightarrow 15$

$k=-13 \rightarrow 15$

$l=-16 \rightarrow 16$

Hydrogen site location: mixed

$\mathrm{H}$ atoms treated by a mixture of independent and constrained refinement

$w=1 /\left[\sigma^{2}\left(F_{\mathrm{o}}{ }^{2}\right)+(0.090 P)^{2}\right]$

where $P=\left(F_{\mathrm{o}}^{2}+2 F_{\mathrm{c}}^{2}\right) / 3$

$(\Delta / \sigma)_{\max }=0.001$

$\Delta \rho_{\max }=0.35$ e $\AA^{-3}$

$\Delta \rho_{\text {min }}=-0.32$ e $\AA^{-3}$

Extinction correction: SHELXL-2014/7, $\mathrm{Fc}^{*}=\mathrm{kFc}\left[1+0.001 \times \mathrm{Fc}^{2} \lambda^{3} / \sin (2 \theta)\right]^{-1 / 4}$

Extinction coefficient: 0.019 (4) 
Fractional atomic coordinates and isotropic or equivalent isotropic displacement parameters $\left(\AA^{2}\right)$

\begin{tabular}{|c|c|c|c|c|}
\hline & $x$ & $y$ & $z$ & $U_{\text {iso }} * / U_{\text {eq }}$ \\
\hline $\mathrm{O} 1$ & $0.21741(6)$ & $0.84171(6)$ & $-0.00993(6)$ & $0.02370(16)$ \\
\hline $\mathrm{O} 2$ & $0.26015(7)$ & $0.62711(6)$ & $0.01082(6)$ & $0.02497(16)$ \\
\hline $\mathrm{O} 3$ & $-0.31475(6)$ & $0.79522(6)$ & $-0.19102(6)$ & $0.02084(15)$ \\
\hline $\mathrm{O} 4$ & $1.32849(7)$ & $0.66282(6)$ & $0.36794(6)$ & $0.02574(16)$ \\
\hline O5 & $1.28863(7)$ & $0.87599(6)$ & $0.33722(6)$ & $0.02456(15)$ \\
\hline O6 & $1.86048(6)$ & $0.70388(6)$ & $0.55057(5)$ & $0.01982(14)$ \\
\hline N1 & $0.44816(7)$ & $0.80971(7)$ & $0.08178(6)$ & $0.01957(16)$ \\
\hline N2 & $1.08835(7)$ & $0.69161(7)$ & $0.29159(6)$ & $0.01975(16)$ \\
\hline $\mathrm{C} 1$ & $0.05619(8)$ & $0.74854(8)$ & $-0.06643(7)$ & $0.01587(16)$ \\
\hline $\mathrm{C} 2$ & $-0.02538(8)$ & $0.86942(8)$ & $-0.09987(7)$ & $0.01713(16)$ \\
\hline $\mathrm{H} 2$ & 0.0038 & 0.9435 & -0.0934 & $0.021 *$ \\
\hline $\mathrm{C} 3$ & $-0.14819(8)$ & $0.88217(8)$ & $-0.14234(7)$ & $0.01796(17)$ \\
\hline H3 & -0.2024 & 0.9644 & -0.1660 & $0.022 *$ \\
\hline $\mathrm{C} 4$ & $-0.19207(8)$ & $0.77306(8)$ & $-0.15022(7)$ & $0.01676(17)$ \\
\hline $\mathrm{C} 5$ & $-0.11202(8)$ & $0.65191(8)$ & $-0.11692(7)$ & $0.01732(16)$ \\
\hline H5 & -0.1416 & 0.5779 & -0.1222 & $0.021 *$ \\
\hline C6 & $0.01143(8)$ & $0.64099(8)$ & $-0.07598(7)$ & $0.01671(16)$ \\
\hline H6 & 0.0665 & 0.5586 & -0.0541 & $0.020 *$ \\
\hline $\mathrm{C} 7$ & $0.18746(8)$ & $0.73163(8)$ & $-0.01851(7)$ & $0.01780(17)$ \\
\hline $\mathrm{C} 8$ & $-0.36390(8)$ & $0.68649(8)$ & $-0.20224(7)$ & $0.01802(17)$ \\
\hline H8A & -0.3609 & 0.6385 & -0.1270 & $0.022 *$ \\
\hline H8B & -0.3108 & 0.6283 & -0.2576 & $0.022 *$ \\
\hline $\mathrm{C} 9$ & $-0.50170(9)$ & $0.73524(9)$ & $-0.24460(8)$ & $0.02173(18)$ \\
\hline H9B & -0.5034 & 0.7767 & -0.3228 & $0.026^{*}$ \\
\hline H9A & -0.5522 & 0.7999 & -0.1931 & $0.026^{*}$ \\
\hline $\mathrm{C} 10$ & $-0.56236(9)$ & $0.62384(9)$ & $-0.24724(10)$ & $0.0281(2)$ \\
\hline $\mathrm{H} 10 \mathrm{~A}$ & -0.6465 & 0.6532 & -0.2862 & $0.042 *$ \\
\hline H10B & -0.5742 & 0.5925 & -0.1684 & $0.042 *$ \\
\hline $\mathrm{H} 10 \mathrm{C}$ & -0.5053 & 0.5544 & -0.2889 & $0.042 *$ \\
\hline $\mathrm{C} 11$ & $1.49360(8)$ & $0.75522(8)$ & $0.41394(7)$ & $0.01674(16)$ \\
\hline $\mathrm{C} 12$ & $1.57341(9)$ & $0.63400(8)$ & $0.44879(7)$ & $0.01858(17)$ \\
\hline $\mathrm{H} 12$ & 1.5434 & 0.5605 & 0.4418 & $0.022 *$ \\
\hline C13 & $1.69517(9)$ & $0.62010(8)$ & $0.49316(7)$ & $0.01892(17)$ \\
\hline H13 & 1.7485 & 0.5373 & 0.5164 & $0.023 *$ \\
\hline $\mathrm{C} 14$ & $1.74023(8)$ & $0.72791(8)$ & $0.50403(7)$ & $0.01712(17)$ \\
\hline $\mathrm{C} 15$ & $1.66235(9)$ & $0.84947(8)$ & $0.46796(7)$ & $0.01804(17)$ \\
\hline H15 & 1.6927 & 0.9229 & 0.4740 & $0.022 *$ \\
\hline $\mathrm{C} 16$ & $1.54008(8)$ & $0.86179(8)$ & $0.42315(7)$ & $0.01771(17)$ \\
\hline H16 & 1.4873 & 0.9443 & 0.3984 & $0.021^{*}$ \\
\hline $\mathrm{C} 17$ & $1.36100(9)$ & $0.77237(8)$ & $0.36890(7)$ & $0.01841(17)$ \\
\hline $\mathrm{C} 18$ & $1.91667(8)$ & $0.80929(8)$ & $0.55338(7)$ & $0.01815(17)$ \\
\hline H18A & 1.8635 & 0.8761 & 0.6016 & $0.022 *$ \\
\hline H18B & 1.9206 & 0.8477 & 0.4747 & $0.022 *$ \\
\hline C19 & $2.05137(9)$ & $0.75818(9)$ & $0.60313(8)$ & $0.02037(18)$ \\
\hline H19B & 2.1028 & 0.6888 & 0.5564 & $0.024 *$ \\
\hline
\end{tabular}




$\begin{array}{lllll}\text { H19A } & 2.0465 & 0.7220 & 0.6825 & 0.024^{*} \\ \text { C20 } & 2.11746(9) & 0.86578(9) & 0.60422(9) & 0.0277(2) \\ \text { H20A } & 2.0643 & 0.9360 & 0.6475 & 0.042^{*} \\ \text { H20B } & 2.1277 & 0.8972 & 0.5251 & 0.042^{*} \\ \text { H20C } & 2.2027 & 0.8330 & 0.6408 & 0.042^{*} \\ \text { C21 } & 0.52853(9) & 0.69053(9) & 0.08870(8) & 0.02069(18) \\ \text { H21 } & 0.4970 & 0.6220 & 0.0666 & 0.025^{*} \\ \text { C22 } & 0.65531(8) & 0.66285(8) & 0.12658(8) & 0.01907(17) \\ \text { H22 } & 0.7088 & 0.5771 & 0.1302 & 0.023^{*} \\ \text { C23 } & 0.70378(8) & 0.76194(8) & 0.15928(7) & 0.01608(16) \\ \text { C24 } & 0.61977(8) & 0.88583(8) & 0.15197(7) & 0.01863(17) \\ \text { H24 } & 0.6488 & 0.9563 & 0.1730 & 0.022^{*} \\ \text { C25 } & 0.49411(8) & 0.90534(8) & 0.11387(7) & 0.01989(17) \\ \text { H25 } & 0.4380 & 0.9900 & 0.1103 & 0.024^{*} \\ \text { C26 } & 1.02841(8) & 0.80376(8) & 0.23616(7) & 0.02020(18) \\ \text { H26 } & 1.0730 & 0.8678 & 0.2273 & 0.024^{*} \\ \text { C27 } & 0.90438(8) & 0.83033(8) & 0.19124(7) & 0.01839(17) \\ \text { H27 } & 0.8655 & 0.9110 & 0.1527 & 0.022^{*} \\ \text { C28 } & 0.83731(8) & 0.73745(8) & 0.20317(7) & 0.01565(16) \\ \text { C29 } & 0.90043(8) & 0.62059(8) & 0.26043(7) & 0.01836(17) \\ \text { H29 } & 0.8586 & 0.5544 & 0.2701 & 0.022^{*} \\ \text { C30 } & 1.02416(9) & 0.60203(8) & 0.30285(7) & 0.02023(18) \\ \text { H30 } & 1.0656 & 0.5222 & 0.3417 & 0.024^{*} \\ \text { H1 } & 0.305(2) & 0.8292(19) & 0.0308(18) & 0.088(6)^{*} \\ \text { H4 } & 1.240(2) & 0.6740(19) & 0.3337(16) & 0.082(6)^{*} \\ & & & & \end{array}$

Atomic displacement parameters $\left(\AA^{2}\right)$

\begin{tabular}{lllllll}
\hline & $U^{11}$ & $U^{22}$ & $U^{33}$ & $U^{12}$ & $U^{13}$ & $U^{23}$ \\
\hline O1 & $0.0188(3)$ & $0.0171(3)$ & $0.0356(4)$ & $-0.0057(3)$ & $-0.0076(3)$ & $0.0000(3)$ \\
O2 & $0.0195(3)$ & $0.0176(3)$ & $0.0355(4)$ & $-0.0016(2)$ & $-0.0077(3)$ & $0.0014(3)$ \\
O3 & $0.0148(3)$ & $0.0155(3)$ & $0.0318(3)$ & $-0.0040(2)$ & $-0.0071(2)$ & $0.0017(2)$ \\
O4 & $0.0198(3)$ & $0.0180(3)$ & $0.0396(4)$ & $-0.0060(3)$ & $-0.0084(3)$ & $0.0027(3)$ \\
O5 & $0.0202(3)$ & $0.0193(3)$ & $0.0314(3)$ & $-0.0018(3)$ & $-0.0051(3)$ & $0.0043(3)$ \\
O6 & $0.0175(3)$ & $0.0163(3)$ & $0.0255(3)$ & $-0.0048(2)$ & $-0.0064(2)$ & $0.0022(2)$ \\
N1 & $0.0156(3)$ & $0.0211(4)$ & $0.0216(3)$ & $-0.0048(3)$ & $-0.0021(3)$ & $0.0009(3)$ \\
N2 & $0.0166(3)$ & $0.0199(3)$ & $0.0219(3)$ & $-0.0035(3)$ & $-0.0022(3)$ & $-0.0019(3)$ \\
C1 & $0.0154(4)$ & $0.0154(4)$ & $0.0163(3)$ & $-0.0036(3)$ & $-0.0007(3)$ & $0.0006(3)$ \\
C2 & $0.0171(4)$ & $0.0139(3)$ & $0.0207(4)$ & $-0.0049(3)$ & $-0.0016(3)$ & $0.0000(3)$ \\
C3 & $0.0165(4)$ & $0.0132(3)$ & $0.0229(4)$ & $-0.0025(3)$ & $-0.0028(3)$ & $0.0018(3)$ \\
C4 & $0.0143(4)$ & $0.0161(4)$ & $0.0188(4)$ & $-0.0028(3)$ & $-0.0019(3)$ & $0.0007(3)$ \\
C5 & $0.0178(4)$ & $0.0139(4)$ & $0.0205(4)$ & $-0.0049(3)$ & $-0.0018(3)$ & $0.0003(3)$ \\
C6 & $0.0168(4)$ & $0.0134(3)$ & $0.0186(3)$ & $-0.0024(3)$ & $-0.0017(3)$ & $0.0012(3)$ \\
C7 & $0.0167(4)$ & $0.0173(4)$ & $0.0190(4)$ & $-0.0041(3)$ & $-0.0011(3)$ & $-0.0008(3)$ \\
C8 & $0.0172(4)$ & $0.0164(4)$ & $0.0206(4)$ & $-0.0051(3)$ & $-0.0024(3)$ & $0.0003(3)$ \\
C9 & $0.0169(4)$ & $0.0194(4)$ & $0.0287(4)$ & $-0.0052(3)$ & $-0.0052(3)$ & $0.0013(3)$ \\
C10 & $0.0205(4)$ & $0.0241(4)$ & $0.0409(5)$ & $-0.0080(4)$ & $-0.0064(4)$ & $-0.0010(4)$ \\
C11 & $0.0161(4)$ & $0.0166(4)$ & $0.0168(3)$ & $-0.0036(3)$ & $-0.0004(3)$ & $0.0003(3)$
\end{tabular}




$\begin{array}{lllllll}\mathrm{C} 12 & 0.0196(4) & 0.0153(4) & 0.0209(4) & -0.0052(3) & -0.0017(3) & 0.0005(3) \\ \mathrm{C} 13 & 0.0196(4) & 0.0136(4) & 0.0220(4) & -0.0024(3) & -0.0031(3) & 0.0017(3) \\ \mathrm{C} 14 & 0.0163(4) & 0.0168(4) & 0.0170(3) & -0.0027(3) & -0.0011(3) & 0.0005(3) \\ \mathrm{C} 15 & 0.0189(4) & 0.0146(4) & 0.0203(4) & -0.0042(3) & -0.0012(3) & 0.0000(3) \\ \text { C16 } & 0.0177(4) & 0.0148(4) & 0.0187(4) & -0.0016(3) & -0.0006(3) & 0.0010(3) \\ \text { C17 } & 0.0176(4) & 0.0185(4) & 0.0179(4) & -0.0034(3) & -0.0003(3) & 0.0006(3) \\ \text { C18 } & 0.0178(4) & 0.0161(4) & 0.0206(4) & -0.0051(3) & -0.0021(3) & 0.0010(3) \\ \text { C19 } & 0.0177(4) & 0.0193(4) & 0.0239(4) & -0.0052(3) & -0.0035(3) & 0.0017(3) \\ \text { C20 } & 0.0213(4) & 0.0252(4) & 0.0376(5) & -0.0091(4) & -0.0056(4) & 0.0043(4) \\ \text { C21 } & 0.0184(4) & 0.0204(4) & 0.0240(4) & -0.0064(3) & -0.0024(3) & -0.0019(3) \\ \text { C22 } & 0.0163(4) & 0.0164(4) & 0.0238(4) & -0.0031(3) & -0.0019(3) & -0.0010(3) \\ \text { C23 } & 0.0142(4) & 0.0172(4) & 0.0160(3) & -0.0035(3) & 0.0002(3) & 0.0010(3) \\ \text { C24 } & 0.0174(4) & 0.0158(4) & 0.0223(4) & -0.0042(3) & -0.0012(3) & -0.0003(3) \\ \text { C25 } & 0.0164(4) & 0.0180(4) & 0.0235(4) & -0.0025(3) & -0.0016(3) & 0.0025(3) \\ \text { C26 } & 0.0171(4) & 0.0214(4) & 0.0227(4) & -0.0066(3) & -0.0014(3) & 0.0006(3) \\ \text { C27 } & 0.0167(4) & 0.0167(4) & 0.0213(4) & -0.0043(3) & -0.0016(3) & 0.0018(3) \\ \text { C28 } & 0.0138(4) & 0.0164(4) & 0.0161(3) & -0.0030(3) & 0.0000(3) & -0.0017(3) \\ \text { C29 } & 0.0169(4) & 0.0157(4) & 0.0219(4) & -0.0036(3) & -0.0018(3) & -0.0002(3) \\ \text { C30 } & 0.0180(4) & 0.0175(4) & 0.0235(4) & -0.0021(3) & -0.0037(3) & -0.0002(3)\end{array}$

Geometric parameters $\left(\AA,{ }^{\circ}\right)$

\begin{tabular}{llll}
\hline $\mathrm{O} 1-\mathrm{C} 7$ & $1.3335(10)$ & $\mathrm{C} 11-\mathrm{C} 17$ & $1.4853(12)$ \\
$\mathrm{O} 1-\mathrm{H} 1$ & $1.03(2)$ & $\mathrm{C} 12-\mathrm{C} 13$ & $1.3802(12)$ \\
$\mathrm{O} 2-\mathrm{C} 7$ & $1.2208(11)$ & $\mathrm{C} 12-\mathrm{H} 12$ & 0.9500 \\
$\mathrm{O} 3-\mathrm{C} 4$ & $1.3612(10)$ & $\mathrm{C} 13-\mathrm{C} 14$ & $1.4010(11)$ \\
$\mathrm{O} 3-\mathrm{C} 8$ & $1.4368(10)$ & $\mathrm{C} 13-\mathrm{H} 13$ & 0.9500 \\
$\mathrm{O} 4-\mathrm{C} 17$ & $1.3331(11)$ & $\mathrm{C} 14-\mathrm{C} 15$ & $1.3993(12)$ \\
$\mathrm{O} 4-\mathrm{H} 4$ & $1.01(2)$ & $\mathrm{C} 15-\mathrm{C} 16$ & $1.3904(12)$ \\
$\mathrm{O} 5-\mathrm{C} 17$ & $1.2190(11)$ & $\mathrm{C} 15-\mathrm{H} 15$ & 0.9500 \\
$\mathrm{O} 6-\mathrm{C} 14$ & $1.3603(10)$ & $\mathrm{C} 16-\mathrm{H} 16$ & 0.9500 \\
$\mathrm{O} 6-\mathrm{C} 18$ & $1.4397(10)$ & $\mathrm{C} 18-\mathrm{C} 19$ & $1.5109(11)$ \\
$\mathrm{N} 1-\mathrm{C} 25$ & $1.3430(11)$ & $\mathrm{C} 18-\mathrm{H} 18 \mathrm{~A}$ & 0.9900 \\
$\mathrm{~N} 1-\mathrm{C} 21$ & $1.3430(12)$ & $\mathrm{C} 18-\mathrm{H} 18 \mathrm{~B}$ & 0.9900 \\
$\mathrm{~N} 2-\mathrm{C} 30$ & $1.3420(11)$ & $\mathrm{C} 19-\mathrm{C} 20$ & $1.5301(12)$ \\
$\mathrm{N} 2-\mathrm{C} 26$ & $1.3433(12)$ & $\mathrm{C} 19-\mathrm{H} 19 \mathrm{~B}$ & 0.9900 \\
$\mathrm{C} 1-\mathrm{C} 6$ & $1.3944(11)$ & $\mathrm{C} 19-\mathrm{H} 19 \mathrm{~A}$ & 0.9900 \\
$\mathrm{C} 1-\mathrm{C} 2$ & $1.4004(12)$ & $\mathrm{C} 20-\mathrm{H} 20 \mathrm{~A}$ & 0.9800 \\
$\mathrm{C} 1-\mathrm{C} 7$ & $1.4848(11)$ & $\mathrm{C} 20-\mathrm{H} 20 \mathrm{~B}$ & 0.9800 \\
$\mathrm{C} 2-\mathrm{C} 3$ & $1.3848(11)$ & $\mathrm{C} 20-\mathrm{H} 20 \mathrm{C}$ & 0.9800 \\
$\mathrm{C} 2-\mathrm{H} 2$ & 0.9500 & $\mathrm{C} 21-\mathrm{C} 22$ & $1.3851(12)$ \\
$\mathrm{C} 3-\mathrm{C} 4$ & $1.4023(11)$ & $\mathrm{C} 21-\mathrm{H} 21$ & 0.9500 \\
$\mathrm{C} 3-\mathrm{H} 3$ & 0.9500 & $\mathrm{C} 22-\mathrm{C} 23$ & $1.3950(11)$ \\
$\mathrm{C} 4-\mathrm{C} 5$ & $1.3963(12)$ & $\mathrm{C} 22-\mathrm{H} 22$ & 0.9500 \\
$\mathrm{C} 5-\mathrm{C} 6$ & $1.3886(11)$ & $\mathrm{C} 23-\mathrm{C} 24$ & $1.3981(11)$ \\
$\mathrm{C} 5-\mathrm{H} 5$ & 0.9500 & $\mathrm{C} 23-\mathrm{C} 28$ & $1.4790(11)$ \\
$\mathrm{C} 6-\mathrm{H} 6$ & 0.9500 & $\mathrm{C} 24-\mathrm{C} 25$ & $1.3840(12)$ \\
$\mathrm{C} 8-\mathrm{C} 9$ & $1.5084(11)$ & $\mathrm{C} 24-\mathrm{H} 24$ & 0.9500 \\
& & &
\end{tabular}




\begin{tabular}{|c|c|c|c|}
\hline $\mathrm{C} 8-\mathrm{H} 8 \mathrm{~A}$ & 0.9900 & $\mathrm{C} 25-\mathrm{H} 25$ & 0.9500 \\
\hline $\mathrm{C} 8-\mathrm{H} 8 \mathrm{~B}$ & 0.9900 & $\mathrm{C} 26-\mathrm{C} 27$ & $1.3875(11)$ \\
\hline $\mathrm{C} 9-\mathrm{C} 10$ & $1.5301(12)$ & $\mathrm{C} 26-\mathrm{H} 26$ & 0.9500 \\
\hline C9-H9B & 0.9900 & $\mathrm{C} 27-\mathrm{C} 28$ & $1.3955(11)$ \\
\hline C9-H9A & 0.9900 & $\mathrm{C} 27-\mathrm{H} 27$ & 0.9500 \\
\hline $\mathrm{C} 10-\mathrm{H} 10 \mathrm{~A}$ & 0.9800 & $\mathrm{C} 28-\mathrm{C} 29$ & $1.3990(12)$ \\
\hline $\mathrm{C} 10-\mathrm{H} 10 \mathrm{~B}$ & 0.9800 & $\mathrm{C} 29-\mathrm{C} 30$ & $1.3834(11)$ \\
\hline $\mathrm{C} 10-\mathrm{H} 10 \mathrm{C}$ & 0.9800 & $\mathrm{C} 29-\mathrm{H} 29$ & 0.9500 \\
\hline $\mathrm{C} 11-\mathrm{C} 16$ & $1.3946(11)$ & $\mathrm{C} 30-\mathrm{H} 30$ & 0.9500 \\
\hline $\mathrm{C} 11-\mathrm{C} 12$ & $1.4002(12)$ & & \\
\hline $\mathrm{C} 7-\mathrm{O} 1-\mathrm{H} 1$ & $112.5(11)$ & $\mathrm{C} 16-\mathrm{C} 15-\mathrm{C} 14$ & $119.37(8)$ \\
\hline $\mathrm{C} 4-\mathrm{O} 3-\mathrm{C} 8$ & $117.72(6)$ & $\mathrm{C} 16-\mathrm{C} 15-\mathrm{H} 15$ & 120.3 \\
\hline $\mathrm{C} 17-\mathrm{O} 4-\mathrm{H} 4$ & $113.0(11)$ & $\mathrm{C} 14-\mathrm{C} 15-\mathrm{H} 15$ & 120.3 \\
\hline $\mathrm{C} 14-\mathrm{O} 6-\mathrm{C} 18$ & $117.65(6)$ & $\mathrm{C} 15-\mathrm{C} 16-\mathrm{C} 11$ & $121.16(8)$ \\
\hline $\mathrm{C} 25-\mathrm{N} 1-\mathrm{C} 21$ & $117.73(7)$ & $\mathrm{C} 15-\mathrm{C} 16-\mathrm{H} 16$ & 119.4 \\
\hline $\mathrm{C} 30-\mathrm{N} 2-\mathrm{C} 26$ & $117.69(7)$ & $\mathrm{C} 11-\mathrm{C} 16-\mathrm{H} 16$ & 119.4 \\
\hline $\mathrm{C} 6-\mathrm{C} 1-\mathrm{C} 2$ & $118.87(7)$ & $\mathrm{O} 5-\mathrm{C} 17-\mathrm{O} 4$ & $123.34(8)$ \\
\hline $\mathrm{C} 6-\mathrm{C} 1-\mathrm{C} 7$ & $119.01(7)$ & $\mathrm{O} 5-\mathrm{C} 17-\mathrm{C} 11$ & $123.60(8)$ \\
\hline $\mathrm{C} 2-\mathrm{C} 1-\mathrm{C} 7$ & $122.11(7)$ & $\mathrm{O} 4-\mathrm{C} 17-\mathrm{C} 11$ & $113.06(7)$ \\
\hline $\mathrm{C} 3-\mathrm{C} 2-\mathrm{C} 1$ & $120.70(7)$ & $\mathrm{O} 6-\mathrm{C} 18-\mathrm{C} 19$ & $107.89(7)$ \\
\hline $\mathrm{C} 3-\mathrm{C} 2-\mathrm{H} 2$ & 119.6 & $\mathrm{O} 6-\mathrm{C} 18-\mathrm{H} 18 \mathrm{~A}$ & 110.1 \\
\hline $\mathrm{C} 1-\mathrm{C} 2-\mathrm{H} 2$ & 119.6 & $\mathrm{C} 19-\mathrm{C} 18-\mathrm{H} 18 \mathrm{~A}$ & 110.1 \\
\hline $\mathrm{C} 2-\mathrm{C} 3-\mathrm{C} 4$ & $119.63(7)$ & $\mathrm{O} 6-\mathrm{C} 18-\mathrm{H} 18 \mathrm{~B}$ & 110.1 \\
\hline $\mathrm{C} 2-\mathrm{C} 3-\mathrm{H} 3$ & 120.2 & $\mathrm{C} 19-\mathrm{C} 18-\mathrm{H} 18 \mathrm{~B}$ & 110.1 \\
\hline $\mathrm{C} 4-\mathrm{C} 3-\mathrm{H} 3$ & 120.2 & $\mathrm{H} 18 \mathrm{~A}-\mathrm{C} 18-\mathrm{H} 18 \mathrm{~B}$ & 108.4 \\
\hline $\mathrm{O} 3-\mathrm{C} 4-\mathrm{C} 5$ & $124.21(8)$ & $\mathrm{C} 18-\mathrm{C} 19-\mathrm{C} 20$ & $110.05(7)$ \\
\hline $\mathrm{O} 3-\mathrm{C} 4-\mathrm{C} 3$ & $115.45(7)$ & $\mathrm{C} 18-\mathrm{C} 19-\mathrm{H} 19 \mathrm{~B}$ & 109.6 \\
\hline $\mathrm{C} 5-\mathrm{C} 4-\mathrm{C} 3$ & $120.34(8)$ & $\mathrm{C} 20-\mathrm{C} 19-\mathrm{H} 19 \mathrm{~B}$ & 109.6 \\
\hline $\mathrm{C} 6-\mathrm{C} 5-\mathrm{C} 4$ & $119.14(8)$ & $\mathrm{C} 18-\mathrm{C} 19-\mathrm{H} 19 \mathrm{~A}$ & 109.6 \\
\hline $\mathrm{C} 6-\mathrm{C} 5-\mathrm{H} 5$ & 120.4 & $\mathrm{C} 20-\mathrm{C} 19-\mathrm{H} 19 \mathrm{~A}$ & 109.6 \\
\hline $\mathrm{C} 4-\mathrm{C} 5-\mathrm{H} 5$ & 120.4 & $\mathrm{H} 19 \mathrm{~B}-\mathrm{C} 19-\mathrm{H} 19 \mathrm{~A}$ & 108.2 \\
\hline $\mathrm{C} 5-\mathrm{C} 6-\mathrm{C} 1$ & $121.31(8)$ & $\mathrm{C} 19-\mathrm{C} 20-\mathrm{H} 20 \mathrm{~A}$ & 109.5 \\
\hline $\mathrm{C} 5-\mathrm{C} 6-\mathrm{H} 6$ & 119.3 & $\mathrm{C} 19-\mathrm{C} 20-\mathrm{H} 20 \mathrm{~B}$ & 109.5 \\
\hline $\mathrm{C} 1-\mathrm{C} 6-\mathrm{H} 6$ & 119.3 & $\mathrm{H} 20 \mathrm{~A}-\mathrm{C} 20-\mathrm{H} 20 \mathrm{~B}$ & 109.5 \\
\hline $\mathrm{O} 2-\mathrm{C} 7-\mathrm{O} 1$ & $123.50(8)$ & $\mathrm{C} 19-\mathrm{C} 20-\mathrm{H} 20 \mathrm{C}$ & 109.5 \\
\hline $\mathrm{O} 2-\mathrm{C} 7-\mathrm{C} 1$ & $123.11(8)$ & $\mathrm{H} 20 \mathrm{~A}-\mathrm{C} 20-\mathrm{H} 20 \mathrm{C}$ & 109.5 \\
\hline $\mathrm{O} 1-\mathrm{C} 7-\mathrm{C} 1$ & $113.39(7)$ & $\mathrm{H} 20 \mathrm{~B}-\mathrm{C} 20-\mathrm{H} 20 \mathrm{C}$ & 109.5 \\
\hline $\mathrm{O} 3-\mathrm{C} 8-\mathrm{C} 9$ & $107.90(7)$ & $\mathrm{N} 1-\mathrm{C} 21-\mathrm{C} 22$ & $123.03(8)$ \\
\hline $\mathrm{O} 3-\mathrm{C} 8-\mathrm{H} 8 \mathrm{~A}$ & 110.1 & $\mathrm{~N} 1-\mathrm{C} 21-\mathrm{H} 21$ & 118.5 \\
\hline $\mathrm{C} 9-\mathrm{C} 8-\mathrm{H} 8 \mathrm{~A}$ & 110.1 & $\mathrm{C} 22-\mathrm{C} 21-\mathrm{H} 21$ & 118.5 \\
\hline $\mathrm{O} 3-\mathrm{C} 8-\mathrm{H} 8 \mathrm{~B}$ & 110.1 & $\mathrm{C} 21-\mathrm{C} 22-\mathrm{C} 23$ & $119.47(8)$ \\
\hline $\mathrm{C} 9-\mathrm{C} 8-\mathrm{H} 8 \mathrm{~B}$ & 110.1 & $\mathrm{C} 21-\mathrm{C} 22-\mathrm{H} 22$ & 120.3 \\
\hline $\mathrm{H} 8 \mathrm{~A}-\mathrm{C} 8-\mathrm{H} 8 \mathrm{~B}$ & 108.4 & $\mathrm{C} 23-\mathrm{C} 22-\mathrm{H} 22$ & 120.3 \\
\hline $\mathrm{C} 8-\mathrm{C} 9-\mathrm{C} 10$ & $109.79(7)$ & $\mathrm{C} 22-\mathrm{C} 23-\mathrm{C} 24$ & $117.30(8)$ \\
\hline $\mathrm{C} 8-\mathrm{C} 9-\mathrm{H} 9 \mathrm{~B}$ & 109.7 & $\mathrm{C} 22-\mathrm{C} 23-\mathrm{C} 28$ & $121.74(8)$ \\
\hline $\mathrm{C} 10-\mathrm{C} 9-\mathrm{H} 9 \mathrm{~B}$ & 109.7 & $\mathrm{C} 24-\mathrm{C} 23-\mathrm{C} 28$ & $120.94(8)$ \\
\hline $\mathrm{C} 8-\mathrm{C} 9-\mathrm{H} 9 \mathrm{~A}$ & 109.7 & $\mathrm{C} 25-\mathrm{C} 24-\mathrm{C} 23$ & $119.67(8)$ \\
\hline
\end{tabular}




\begin{tabular}{|c|c|}
\hline $\mathrm{C} 10-\mathrm{C} 9-\mathrm{H} 9 \mathrm{~A}$ & 109.7 \\
\hline $\mathrm{H} 9 \mathrm{~B}-\mathrm{C} 9-\mathrm{H} 9 \mathrm{~A}$ & 108.2 \\
\hline $\mathrm{C} 9-\mathrm{C} 10-\mathrm{H} 10 \mathrm{~A}$ & 109.5 \\
\hline C9- $\mathrm{C} 10-\mathrm{H} 10 \mathrm{~B}$ & 109.5 \\
\hline $\mathrm{H} 10 \mathrm{~A}-\mathrm{C} 10-\mathrm{H} 10 \mathrm{~B}$ & 109.5 \\
\hline $\mathrm{C} 9-\mathrm{C} 10-\mathrm{H} 10 \mathrm{C}$ & 109.5 \\
\hline $\mathrm{H} 10 \mathrm{~A}-\mathrm{C} 10-\mathrm{H} 10 \mathrm{C}$ & 109.5 \\
\hline $\mathrm{H} 10 \mathrm{~B}-\mathrm{C} 10-\mathrm{H} 10 \mathrm{C}$ & 109.5 \\
\hline $\mathrm{C} 16-\mathrm{C} 11-\mathrm{C} 12$ & $118.80(8)$ \\
\hline $\mathrm{C} 16-\mathrm{C} 11-\mathrm{C} 17$ & $119.71(7)$ \\
\hline $\mathrm{C} 12-\mathrm{C} 11-\mathrm{C} 17$ & $121.49(8)$ \\
\hline $\mathrm{C} 13-\mathrm{C} 12-\mathrm{C} 11$ & $120.75(8)$ \\
\hline $\mathrm{C} 13-\mathrm{C} 12-\mathrm{H} 12$ & 119.6 \\
\hline $\mathrm{C} 11-\mathrm{C} 12-\mathrm{H} 12$ & 119.6 \\
\hline $\mathrm{C} 12-\mathrm{C} 13-\mathrm{C} 14$ & $120.08(8)$ \\
\hline $\mathrm{C} 12-\mathrm{C} 13-\mathrm{H} 13$ & 120.0 \\
\hline $\mathrm{C} 14-\mathrm{C} 13-\mathrm{H} 13$ & 120.0 \\
\hline $\mathrm{O} 6-\mathrm{C} 14-\mathrm{C} 15$ & $124.82(7)$ \\
\hline $\mathrm{O} 6-\mathrm{C} 14-\mathrm{C} 13$ & $115.36(7)$ \\
\hline $\mathrm{C} 15-\mathrm{C} 14-\mathrm{C} 13$ & $119.82(8)$ \\
\hline $\mathrm{C} 6-\mathrm{C} 1-\mathrm{C} 2-\mathrm{C} 3$ & $-0.36(13)$ \\
\hline $\mathrm{C} 7-\mathrm{C} 1-\mathrm{C} 2-\mathrm{C} 3$ & $-178.83(7)$ \\
\hline $\mathrm{C} 1-\mathrm{C} 2-\mathrm{C} 3-\mathrm{C} 4$ & $1.02(13)$ \\
\hline $\mathrm{C} 8-\mathrm{O} 3-\mathrm{C} 4-\mathrm{C} 5$ & $-1.09(12)$ \\
\hline $\mathrm{C} 8-\mathrm{O} 3-\mathrm{C} 4-\mathrm{C} 3$ & $179.21(7)$ \\
\hline $\mathrm{C} 2-\mathrm{C} 3-\mathrm{C} 4-\mathrm{O} 3$ & $178.88(7)$ \\
\hline $\mathrm{C} 2-\mathrm{C} 3-\mathrm{C} 4-\mathrm{C} 5$ & $-0.84(13)$ \\
\hline $\mathrm{O} 3-\mathrm{C} 4-\mathrm{C} 5-\mathrm{C} 6$ & $-179.70(7)$ \\
\hline $\mathrm{C} 3-\mathrm{C} 4-\mathrm{C} 5-\mathrm{C} 6$ & $-0.01(13)$ \\
\hline $\mathrm{C} 4-\mathrm{C} 5-\mathrm{C} 6-\mathrm{C} 1$ & $0.69(13)$ \\
\hline $\mathrm{C} 2-\mathrm{C} 1-\mathrm{C} 6-\mathrm{C} 5$ & $-0.51(13)$ \\
\hline $\mathrm{C} 7-\mathrm{C} 1-\mathrm{C} 6-\mathrm{C} 5$ & $178.01(7)$ \\
\hline $\mathrm{C} 6-\mathrm{C} 1-\mathrm{C} 7-\mathrm{O} 2$ & $1.75(13)$ \\
\hline $\mathrm{C} 2-\mathrm{C} 1-\mathrm{C} 7-\mathrm{O} 2$ & $-179.78(8)$ \\
\hline $\mathrm{C} 6-\mathrm{C} 1-\mathrm{C} 7-\mathrm{O} 1$ & $-177.75(7)$ \\
\hline $\mathrm{C} 2-\mathrm{C} 1-\mathrm{C} 7-\mathrm{O} 1$ & $0.72(12)$ \\
\hline $\mathrm{C} 4-\mathrm{O} 3-\mathrm{C} 8-\mathrm{C} 9$ & $178.04(7)$ \\
\hline $\mathrm{O} 3-\mathrm{C} 8-\mathrm{C} 9-\mathrm{C} 10$ & $-174.77(7)$ \\
\hline $\mathrm{C} 16-\mathrm{C} 11-\mathrm{C} 12-\mathrm{C} 13$ & $0.99(13)$ \\
\hline $\mathrm{C} 17-\mathrm{C} 11-\mathrm{C} 12-\mathrm{C} 13$ & $-178.35(8)$ \\
\hline $\mathrm{C} 11-\mathrm{C} 12-\mathrm{C} 13-\mathrm{C} 14$ & $0.13(13)$ \\
\hline $\mathrm{C} 18-\mathrm{O} 6-\mathrm{C} 14-\mathrm{C} 15$ & $-5.96(12)$ \\
\hline $\mathrm{C} 18-\mathrm{O} 6-\mathrm{C} 14-\mathrm{C} 13$ & $174.06(7)$ \\
\hline $\mathrm{C} 12-\mathrm{C} 13-\mathrm{C} 14-\mathrm{O} 6$ & $178.89(7)$ \\
\hline $\mathrm{C} 12-\mathrm{C} 13-\mathrm{C} 14-\mathrm{C} 15$ & $-1.09(13)$ \\
\hline $\mathrm{O} 6-\mathrm{C} 14-\mathrm{C} 15-\mathrm{C} 16$ & $-179.08(7)$ \\
\hline $\mathrm{C} 13-\mathrm{C} 14-\mathrm{C} 15-\mathrm{C} 16$ & $0.91(13)$ \\
\hline
\end{tabular}

$\begin{array}{ll}\mathrm{C} 25-\mathrm{C} 24-\mathrm{H} 24 & 120.2 \\ \mathrm{C} 23-\mathrm{C} 24-\mathrm{H} 24 & 120.2 \\ \mathrm{~N} 1-\mathrm{C} 25-\mathrm{C} 24 & 122.80(8) \\ \mathrm{N} 1-\mathrm{C} 25-\mathrm{H} 25 & 118.6 \\ \mathrm{C} 24-\mathrm{C} 25-\mathrm{H} 25 & 118.6 \\ \mathrm{~N} 2-\mathrm{C} 26-\mathrm{C} 27 & 123.00(8) \\ \mathrm{N} 2-\mathrm{C} 26-\mathrm{H} 26 & 118.5 \\ \mathrm{C} 27-\mathrm{C} 26-\mathrm{H} 26 & 118.5 \\ \mathrm{C} 26-\mathrm{C} 27-\mathrm{C} 28 & 119.40(8) \\ \mathrm{C} 26-\mathrm{C} 27-\mathrm{H} 27 & 120.3 \\ \mathrm{C} 28-\mathrm{C} 27-\mathrm{H} 27 & 120.3 \\ \mathrm{C} 27-\mathrm{C} 28-\mathrm{C} 29 & 117.37(7) \\ \mathrm{C} 27-\mathrm{C} 28-\mathrm{C} 23 & 121.57(8) \\ \mathrm{C} 29-\mathrm{C} 28-\mathrm{C} 23 & 121.05(8) \\ \mathrm{C} 30-\mathrm{C} 29-\mathrm{C} 28 & 119.54(8) \\ \mathrm{C} 30-\mathrm{C} 29-\mathrm{H} 29 & 120.2 \\ \mathrm{C} 28-\mathrm{C} 29-\mathrm{H} 29 & 120.2 \\ \mathrm{~N} 2-\mathrm{C} 30-\mathrm{C} 29 & 123.00(8) \\ \mathrm{N} 2-\mathrm{C} 30-\mathrm{H} 30 & 118.5 \\ \mathrm{C} 29-\mathrm{C} 30-\mathrm{H} 30 & 118.5\end{array}$

$\mathrm{C} 12-\mathrm{C} 11-\mathrm{C} 16-\mathrm{C} 15-1.18$ (13)

$\mathrm{C} 17-\mathrm{C} 11-\mathrm{C} 16-\mathrm{C} 15 \quad 178.18(7)$

$\mathrm{C} 16-\mathrm{C} 11-\mathrm{C} 17-\mathrm{O} 5 \quad 0.98(13)$

$\mathrm{C} 12-\mathrm{C} 11-\mathrm{C} 17-\mathrm{O} 5 \quad-179.69(8)$

$\mathrm{C} 16-\mathrm{C} 11-\mathrm{C} 17-\mathrm{O} 4 \quad-178.26(7)$

$\mathrm{C} 12-\mathrm{C} 11-\mathrm{C} 17-\mathrm{O} 4 \quad 1.08(12)$

$\mathrm{C} 14-\mathrm{O} 6-\mathrm{C} 18-\mathrm{C} 19 \quad-177.34$ (7)

$\mathrm{O} 6-\mathrm{C} 18-\mathrm{C} 19-\mathrm{C} 20 \quad 178.05(7)$

$\mathrm{C} 25-\mathrm{N} 1-\mathrm{C} 21-\mathrm{C} 22 \quad-0.30(13)$

$\mathrm{N} 1-\mathrm{C} 21-\mathrm{C} 22-\mathrm{C} 23 \quad-0.11(14)$

$\mathrm{C} 21-\mathrm{C} 22-\mathrm{C} 23-\mathrm{C} 24 \quad 0.12(12)$

$\mathrm{C} 21-\mathrm{C} 22-\mathrm{C} 23-\mathrm{C} 28 \quad 178.42(8)$

$\mathrm{C} 22-\mathrm{C} 23-\mathrm{C} 24-\mathrm{C} 25 \quad 0.27(12)$

$\mathrm{C} 28-\mathrm{C} 23-\mathrm{C} 24-\mathrm{C} 25-178.04(8)$

$\mathrm{C} 21-\mathrm{N} 1-\mathrm{C} 25-\mathrm{C} 24 \quad 0.72(13)$

$\mathrm{C} 23-\mathrm{C} 24-\mathrm{C} 25-\mathrm{N} 1 \quad-0.72(13)$

$\mathrm{C} 30-\mathrm{N} 2-\mathrm{C} 26-\mathrm{C} 27 \quad 0.30(13)$

$\mathrm{N} 2-\mathrm{C} 26-\mathrm{C} 27-\mathrm{C} 28 \quad-0.11(13)$

$\mathrm{C} 26-\mathrm{C} 27-\mathrm{C} 28-\mathrm{C} 29 \quad-0.23(12)$

$\mathrm{C} 26-\mathrm{C} 27-\mathrm{C} 28-\mathrm{C} 23 \quad 178.56(8)$

$\mathrm{C} 22-\mathrm{C} 23-\mathrm{C} 28-\mathrm{C} 27 \quad 152.95$ (9)

$\mathrm{C} 24-\mathrm{C} 23-\mathrm{C} 28-\mathrm{C} 27 \quad-28.82(12)$

$\mathrm{C} 22-\mathrm{C} 23-\mathrm{C} 28-\mathrm{C} 29 \quad-28.31$ (12)

$\mathrm{C} 24-\mathrm{C} 23-\mathrm{C} 28-\mathrm{C} 29 \quad 149.93(8)$

$\mathrm{C} 27-\mathrm{C} 28-\mathrm{C} 29-\mathrm{C} 30 \quad 0.37(12)$

$\mathrm{C} 23-\mathrm{C} 28-\mathrm{C} 29-\mathrm{C} 30 \quad-178.42(8)$

$\mathrm{C} 26-\mathrm{N} 2-\mathrm{C} 30-\mathrm{C} 29 \quad-0.15(13)$ 


$\mathrm{C} 14-\mathrm{C} 15-\mathrm{C} 16-\mathrm{C} 11 \quad 0.23(13) \quad \mathrm{C} 28-\mathrm{C} 29-\mathrm{C} 30-\mathrm{N} 2 \quad-0.19(13)$

Hydrogen-bond geometry $\left(\AA,{ }^{\circ}\right)$

$\mathrm{Cg} 1$ and $\mathrm{Cg} 2$ are the centroids of the $\mathrm{C} 1-\mathrm{C} 6$ and $\mathrm{C} 11-\mathrm{C} 16$ rings, respectively.

\begin{tabular}{lllll}
\hline$D-\mathrm{H} \cdots A$ & $D-\mathrm{H}$ & $\mathrm{H} \cdots A$ & $D \cdots A$ & $D-\mathrm{H}^{\cdots} A$ \\
\hline $\mathrm{O} 1-\mathrm{H} 1 \cdots \mathrm{N} 1$ & $1.03(2)$ & $1.61(2)$ & $2.6407(10)$ & $174.3(19)$ \\
$\mathrm{O} 4-\mathrm{H} 4 \cdots \mathrm{N} 2$ & $1.01(2)$ & $1.67(2)$ & $2.6728(11)$ & $173.9(18)$ \\
$\mathrm{C} 3-\mathrm{H} 3 \cdots \mathrm{O} 5^{\mathrm{i}}$ & 0.95 & 2.57 & $3.3981(11)$ & 146 \\
$\mathrm{C} 25-\mathrm{H} 25 \cdots \mathrm{O} 3^{\mathrm{ii}}$ & 0.95 & 2.57 & $3.4581(11)$ & 156 \\
$\mathrm{C} 9-\mathrm{H} 9 B \cdots C g 2^{\mathrm{iii}}$ & 0.99 & 2.84 & $3.6750(1)$ & 142 \\
$\mathrm{C} 19-\mathrm{H} 19 A \cdots C g 1^{\mathrm{iv}}$ & 0.99 & 2.72 & $3.5781(1)$ & 146
\end{tabular}

Symmetry codes: (i) $-x+1,-y+2,-z$; (ii) $-x,-y+2,-z$; (iii) $x-2, y, z-1$; (iv) $x+2, y, z+1$.

(III) 4-n-Butoxybenzoic acid-4,4'-bipyridyl (2/1)

Crystal data

$2 \mathrm{C}_{11} \mathrm{H}_{14} \mathrm{O}_{3} \cdot \mathrm{C}_{10} \mathrm{H}_{8} \mathrm{~N}_{2}$

$M_{r}=544.63$

Triclinic, $P \overline{1}$

$a=7.6645(10) \AA$

$b=8.5087(13) \AA$

$c=22.606(3) \AA$

$\alpha=80.498(3)^{\circ}$

$\beta=86.486(3)^{\circ}$

$\gamma=80.082(3)^{\circ}$

$V=1431.5(4) \AA^{3}$

Data collection

Rigaku R-AXIS RAPIDII diffractometer

Detector resolution: 10.000 pixels $\mathrm{mm}^{-1}$

$\omega$ scans

12433 measured reflections

5612 independent reflections

\section{Refinement}

Refinement on $F^{2}$

Least-squares matrix: full

$R\left[F^{2}>2 \sigma\left(F^{2}\right)\right]=0.069$

$w R\left(F^{2}\right)=0.193$

$S=1.01$

5610 reflections

371 parameters

0 restraints

Primary atom site location: structure-invariant direct methods
$Z=2$

$F(000)=580.00$

$D_{\mathrm{x}}=1.264 \mathrm{Mg} \mathrm{m}^{-3}$

Mo $K \alpha$ radiation, $\lambda=0.71075 \AA$

Cell parameters from 9788 reflections

$\theta=3.2-30.1^{\circ}$

$\mu=0.09 \mathrm{~mm}^{-1}$

$T=93 \mathrm{~K}$

Platelet, colorless

$0.53 \times 0.41 \times 0.11 \mathrm{~mm}$

3432 reflections with $I>2 \sigma(I)$

$R_{\text {int }}=0.075$

$\theta_{\max }=26.0^{\circ}$

$h=-8 \rightarrow 9$

$k=-10 \rightarrow 10$

$l=-27 \rightarrow 27$

Secondary atom site location: difference Fourier map

Hydrogen site location: mixed

$\mathrm{H}$ atoms treated by a mixture of independent and constrained refinement

$w=1 /\left[\sigma^{2}\left(F_{\mathrm{o}}{ }^{2}\right)+(0.0965 P)^{2}\right]$

where $P=\left(F_{\mathrm{o}}^{2}+2 F_{\mathrm{c}}{ }^{2}\right) / 3$

$(\Delta / \sigma)_{\max }<0.001$

$\Delta \rho_{\text {max }}=0.24 \mathrm{e} \AA^{-3}$

$\Delta \rho_{\min }=-0.41$ e $\AA^{-3}$ 


\section{Special details}

Geometry. All esds (except the esd in the dihedral angle between two 1.s. planes) are estimated using the full covariance matrix. The cell esds are taken into account individually in the estimation of esds in distances, angles and torsion angles; correlations between esds in cell parameters are only used when they are defined by crystal symmetry. An approximate (isotropic) treatment of cell esds is used for estimating esds involving l.s. planes.

Refinement. Reflections were merged by SHELXL according to the crystal class for the calculation of statistics and refinement.

Fractional atomic coordinates and isotropic or equivalent isotropic displacement parameters $\left(\AA^{2}\right)$

\begin{tabular}{|c|c|c|c|c|}
\hline & $x$ & $y$ & $z$ & $U_{\text {iso }} * / U_{\text {eq }}$ \\
\hline $\mathrm{O} 1$ & $0.1390(2)$ & $-0.1143(2)$ & $-0.18237(8)$ & $0.0358(4)$ \\
\hline $\mathrm{O} 2$ & $0.3054(2)$ & $-0.3129(2)$ & $-0.12317(8)$ & $0.0399(5)$ \\
\hline $\mathrm{O} 3$ & $0.1632(2)$ & $-0.6711(2)$ & $-0.33646(8)$ & $0.0347(4)$ \\
\hline $\mathrm{O} 4$ & $0.2363(2)$ & $0.7005(2)$ & $0.21058(8)$ & $0.0380(5)$ \\
\hline $\mathrm{O} 5$ & $0.3659(3)$ & $0.8609(3)$ & $0.13954(8)$ & $0.0433(5)$ \\
\hline O6 & $0.3442(2)$ & $1.2321(2)$ & $0.36266(7)$ & $0.0336(4)$ \\
\hline N1 & $0.1708(3)$ & $0.0497(3)$ & $-0.09736(10)$ & $0.0375(5)$ \\
\hline N2 & $0.2511(3)$ & $0.5220(3)$ & $0.12499(9)$ & $0.0343(5)$ \\
\hline $\mathrm{C} 1$ & $0.2120(3)$ & $-0.3677(3)$ & $-0.21459(10)$ & $0.0288(5)$ \\
\hline $\mathrm{C} 2$ & $0.1061(3)$ & $-0.3154(3)$ & $-0.26457(11)$ & $0.0318(6)$ \\
\hline $\mathrm{H} 2$ & 0.0429 & -0.2080 & -0.2710 & $0.038^{*}$ \\
\hline $\mathrm{C} 3$ & $0.0926(3)$ & $-0.4186(3)$ & $-0.30463(11)$ & $0.0325(6)$ \\
\hline H3 & 0.0206 & -0.3821 & -0.3385 & $0.039 *$ \\
\hline $\mathrm{C} 4$ & $0.1843(3)$ & $-0.5760(3)$ & $-0.29530(11)$ & $0.0306(5)$ \\
\hline $\mathrm{C} 5$ & $0.2917(3)$ & $-0.6282(3)$ & $-0.24626(11)$ & $0.0329(6)$ \\
\hline H5 & 0.3555 & -0.7354 & -0.2399 & $0.040^{*}$ \\
\hline C6 & $0.3055(3)$ & $-0.5231(3)$ & $-0.20661(11)$ & $0.0323(6)$ \\
\hline H6 & 0.3804 & -0.5586 & -0.1734 & $0.039 *$ \\
\hline $\mathrm{C} 7$ & $0.2243(3)$ & $-0.2636(3)$ & $-0.16939(11)$ & $0.0323(6)$ \\
\hline $\mathrm{C} 8$ & $0.2479(3)$ & $-0.8367(3)$ & $-0.32529(12)$ & $0.0338(6)$ \\
\hline H8A & 0.2005 & -0.8918 & -0.2872 & $0.041^{*}$ \\
\hline H8B & 0.3771 & -0.8427 & -0.3219 & $0.041^{*}$ \\
\hline C9 & $0.2121(3)$ & $-0.9175(4)$ & $-0.37668(11)$ & $0.0352(6)$ \\
\hline H9A & 0.2542 & -1.0350 & -0.3663 & $0.042 *$ \\
\hline H9B & 0.0824 & -0.9016 & -0.3814 & $0.042 *$ \\
\hline $\mathrm{C} 10$ & $0.2976(3)$ & $-0.8574(4)$ & $-0.43586(12)$ & $0.0381(6)$ \\
\hline $\mathrm{H} 10 \mathrm{~A}$ & 0.4271 & -0.8701 & -0.4313 & $0.046^{*}$ \\
\hline H10B & 0.2521 & -0.7409 & -0.4475 & $0.046^{*}$ \\
\hline C11 & $0.2622(4)$ & $-0.9474(4)$ & $-0.48561(13)$ & $0.0474(7)$ \\
\hline H11A & 0.3074 & -1.0629 & -0.4745 & $0.071 *$ \\
\hline H11B & 0.3221 & -0.9055 & -0.5230 & $0.071^{*}$ \\
\hline $\mathrm{H} 11 \mathrm{C}$ & 0.1343 & -0.9319 & -0.4914 & $0.071^{*}$ \\
\hline $\mathrm{C} 12$ & $0.3175(3)$ & $0.9341(3)$ & $0.23705(11)$ & $0.0300(5)$ \\
\hline $\mathrm{C} 13$ & $0.2420(3)$ & $0.9052(3)$ & $0.29435(11)$ & $0.0300(5)$ \\
\hline H13 & 0.1816 & 0.8155 & 0.3053 & $0.036^{*}$ \\
\hline $\mathrm{C} 14$ & $0.2538(3)$ & $1.0058(3)$ & $0.33557(11)$ & $0.0301(5)$ \\
\hline H14 & 0.2019 & 0.9852 & 0.3747 & $0.036^{*}$ \\
\hline
\end{tabular}




$\begin{array}{lllll}\text { C15 } & 0.3423(3) & 1.1382(3) & 0.31945(11) & 0.0305(5) \\ \text { C16 } & 0.4185(3) & 1.1671(3) & 0.26282(11) & 0.0325(6) \\ \text { H16 } & 0.4781 & 1.2572 & 0.2518 & 0.039^{*} \\ \text { C17 } & 0.4079(3) & 1.0642(3) & 0.22178(11) & 0.0320(6) \\ \text { H17 } & 0.4629 & 1.0829 & 0.1830 & 0.038^{*} \\ \text { C18 } & 0.3088(3) & 0.8303(3) & 0.19068(11) & 0.0323(6) \\ \text { C19 } & 0.4228(3) & 1.3756(3) & 0.34655(12) & 0.0375(6) \\ \text { H19A } & 0.3595 & 1.4478 & 0.3129 & 0.045^{*} \\ \text { H19B } & 0.5485 & 1.3469 & 0.3337 & 0.045^{*} \\ \text { C20 } & 0.4099(3) & 1.4595(4) & 0.40073(12) & 0.0376(6) \\ \text { H20A } & 0.4719 & 1.5539 & 0.3912 & 0.045^{*} \\ \text { H20B } & 0.4720 & 1.3845 & 0.4340 & 0.045^{*} \\ \text { C21 } & 0.2211(3) & 1.5162(4) & 0.42172(12) & 0.0372(6) \\ \text { H21A } & 0.1552 & 1.5829 & 0.3875 & 0.045^{*} \\ \text { H21B } & 0.1624 & 1.4210 & 0.4354 & 0.045^{*} \\ \text { C22 } & 0.2123(4) & 1.6142(4) & 0.47241(13) & 0.0452(7) \\ \text { H22A } & 0.2700 & 1.5464 & 0.5075 & 0.068^{*} \\ \text { H22B } & 0.0881 & 1.6524 & 0.4830 & 0.068^{*} \\ \text { H22C } & 0.2731 & 1.7070 & 0.4596 & 0.068^{*} \\ \text { C23 } & 0.2365(4) & -0.0184(4) & -0.04412(13) & 0.0472(7) \\ \text { H23 } & 0.2711 & -0.1324 & -0.0368 & 0.057^{*} \\ \text { C24 } & 0.2571(4) & 0.0681(4) & 0.00093(12) & 0.0421(7) \\ \text { H24 } & 0.3000 & 0.0144 & 0.0388 & 0.051^{*} \\ \text { C25 } & 0.2135(3) & 0.2354(3) & -0.01037(11) & 0.0307(5) \\ \text { C26 } & 0.1489(3) & 0.3070(3) & -0.06606(11) & 0.0332(6) \\ \text { H26 } & 0.1191 & 0.4211 & -0.0756 & 0.040^{*} \\ \text { C27 } & 0.1286(3) & 0.2094(3) & -0.10754(12) & 0.0347(6) \\ \text { H27 } & 0.0819 & 0.2593 & -0.1453 & 0.042^{*} \\ \text { C28 } & 0.2950(3) & 0.5728(4) & 0.06786(11) & 0.0365(6) \\ \text { H28 } & 0.3359 & 0.6735 & 0.0581 & 0.044^{*} \\ \text { C29 } & 0.2834(3) & 0.4853(3) & 0.02218(11) & 0.0329(6) \\ \text { H29 } & 0.3116 & 0.5271 & -0.0182 & 0.040^{*} \\ \text { C30 } & 0.2296(3) & 0.3348(3) & 0.03651(11) & 0.0298(5) \\ \text { C31 } & 0.1889(3) & 0.2808(3) & 0.09596(11) & 0.0334(6) \\ \text { H31 } & 0.1551 & 0.1775 & 0.1075 & 0.040^{*} \\ \text { C32 } & 0.1978(3) & 0.3784(3) & 0.13857(11) & 0.0332(6) \\ \text { H32 } & 0.1648 & 0.3420 & 0.1790 & 0.040^{*} \\ \text { H1 } & 0.160(4) & -0.054(4) & -0.1475(15) & 0.060(9)^{*} \\ \text { H4 } & 0.242(5) & 0.642(5) & 0.1754(16) & 0.065(10)^{*} \\ & & & & \end{array}$

Atomic displacement parameters $\left(\AA^{2}\right)$

\begin{tabular}{lllllll}
\hline & $U^{11}$ & $U^{22}$ & $U^{33}$ & $U^{12}$ & $U^{13}$ & $U^{23}$ \\
\hline O1 & $0.0412(10)$ & $0.0304(11)$ & $0.0354(10)$ & $-0.0006(8)$ & $-0.0033(8)$ & $-0.0091(8)$ \\
O2 & $0.0485(11)$ & $0.0369(12)$ & $0.0331(10)$ & $0.0009(9)$ & $-0.0080(8)$ & $-0.0085(8)$ \\
O3 & $0.0326(9)$ & $0.0333(11)$ & $0.0377(10)$ & $0.0069(8)$ & $-0.0063(7)$ & $-0.0156(8)$ \\
O4 & $0.0436(10)$ & $0.0351(12)$ & $0.0371(10)$ & $-0.0067(8)$ & $-0.0014(8)$ & $-0.0110(9)$ \\
O5 & $0.0581(12)$ & $0.0399(13)$ & $0.0319(10)$ & $-0.0076(10)$ & $0.0004(9)$ & $-0.0064(9)$
\end{tabular}




\begin{tabular}{|c|c|c|c|c|c|c|}
\hline O6 & $0.0371(9)$ & $0.0322(11)$ & $0.0333(9)$ & $-0.0080(8)$ & $0.0011(7)$ & $-0.0089(8)$ \\
\hline $\mathrm{N} 1$ & $0.0455(12)$ & $0.0327(14)$ & $0.0337(12)$ & $-0.0007(10)$ & $-0.0019(9)$ & $-0.0092(10)$ \\
\hline $\mathrm{N} 2$ & $0.0328(10)$ & $0.0357(14)$ & $0.0340(11)$ & $0.0000(9)$ & $-0.0018(9)$ & $-0.0097(10)$ \\
\hline $\mathrm{C} 1$ & $0.0261(11)$ & $0.0307(15)$ & $0.0292(12)$ & $-0.0020(10)$ & $0.0019(9)$ & $-0.0074(10)$ \\
\hline $\mathrm{C} 2$ & $0.0290(11)$ & $0.0315(15)$ & $0.0338(13)$ & $0.0009(10)$ & $-0.0018(10)$ & $-0.0079(11)$ \\
\hline $\mathrm{C} 3$ & $0.0280(11)$ & $0.0358(16)$ & $0.0321(13)$ & $0.0023(11)$ & $-0.0042(10)$ & $-0.0071(11)$ \\
\hline $\mathrm{C} 4$ & $0.0250(11)$ & $0.0340(15)$ & $0.0327(13)$ & $0.0001(10)$ & $-0.0016(9)$ & $-0.0100(11)$ \\
\hline $\mathrm{C} 5$ & $0.0329(12)$ & $0.0283(15)$ & $0.0357(13)$ & $0.0050(10)$ & $-0.0041(10)$ & $-0.0088(11)$ \\
\hline $\mathrm{C} 6$ & $0.0293(11)$ & $0.0369(16)$ & $0.0304(12)$ & $-0.0021(11)$ & $-0.0018(10)$ & $-0.0078(11)$ \\
\hline $\mathrm{C} 7$ & $0.0324(12)$ & $0.0317(15)$ & $0.0328(13)$ & -0.0040 & $0.0008(10)$ & $-0.0074(11)$ \\
\hline $\mathrm{C} 8$ & $0.0357(12)$ & $0.0288(15)$ & $0.0360(14)$ & $0.0005(11)$ & $0.0002(10)$ & $-0.0088(11)$ \\
\hline $\mathrm{C} 9$ & $0.0351(12)$ & $0.0359(16)$ & $0.0360(13)$ & $-0.0052(11)$ & $0.0022(10)$ & $-0.0115(12)$ \\
\hline $\mathrm{C} 10$ & $0.0383(13)$ & $0.0402(17)$ & $0.0365(14)$ & $-0.0050(12)$ & $0.0019(11)$ & $-0.0111(12)$ \\
\hline $\mathrm{C} 11$ & $0.0518(16)$ & $0.054(2)$ & $0.0385(15)$ & $-0.0066(15)$ & $0.0001(13)$ & $-0.0161(14)$ \\
\hline $\mathrm{C} 12$ & $0.0268(11)$ & $0.0271(14)$ & $0.0351(13)$ & $0.0014(10)$ & $-0.0069(10)$ & $-0.0060(11)$ \\
\hline $\mathrm{C} 13$ & $0.0261(11)$ & $0.0291(14)$ & $0.0331(13)$ & $0.0000(10)$ & $-0.0030(10)$ & $-0.0043(11)$ \\
\hline $\mathrm{C} 14$ & $0.0280(11)$ & $0.0326(15)$ & $0.0296(12)$ & $-0.0038(10)$ & $-0.0021(10)$ & $-0.0053(11)$ \\
\hline $\mathrm{C} 15$ & $0.0265(11)$ & $0.0289(15)$ & $0.0353(13)$ & $-0.0004(10)$ & $-0.0035(10)$ & $-0.0058(11)$ \\
\hline $\mathrm{C} 16$ & $0.0312(12)$ & $0.0315(15)$ & $0.0338(13)$ & $-0.0040(11)$ & $-0.0019(10)$ & $-0.0035(11)$ \\
\hline $\mathrm{C} 17$ & $0.0290(11)$ & $0.0341(15)$ & $0.0309(13)$ & $-0.0009(10)$ & $-0.0023(10)$ & $-0.0037(11)$ \\
\hline $\mathrm{C} 18$ & $0.0333(12)$ & $0.0297(15)$ & $0.0317(13)$ & $0.0019(11)$ & $-0.0065(10)$ & $-0.0035(11)$ \\
\hline C19 & $0.0354(13)$ & $0.0357(17)$ & $0.0442(15)$ & $-0.0095(12)$ & $0.0020(11)$ & $-0.0120(12)$ \\
\hline $\mathrm{C} 20$ & $0.0351(13)$ & $0.0387(17)$ & $0.0419(15)$ & $-0.0062(12)$ & $-0.0037(11)$ & $-0.0135(12)$ \\
\hline $\mathrm{C} 21$ & $0.0377(13)$ & $0.0335(16)$ & $0.0397(14)$ & $0.0009(11)$ & $-0.0032(11)$ & $-0.0101(12)$ \\
\hline $\mathrm{C} 22$ & $0.0491(16)$ & $0.0396(18)$ & $0.0474(16)$ & $-0.0021(13)$ & $0.0005(13)$ & $-0.0149(14)$ \\
\hline $\mathrm{C} 23$ & $0.071(2)$ & $0.0278(16)$ & $0.0404(15)$ & $0.0043(14)$ & $-0.0095(14)$ & $-0.0083(13)$ \\
\hline $\mathrm{C} 24$ & $0.0577(17)$ & $0.0306(16)$ & $0.0344(14)$ & $0.0049(13)$ & $-0.0063(12)$ & $-0.0055(12)$ \\
\hline $\mathrm{C} 25$ & $0.0292(11)$ & $0.0310(15)$ & $0.0314(13)$ & $-0.0004(10)$ & $0.0003(10)$ & $-0.0086(11)$ \\
\hline $\mathrm{C} 26$ & $0.0368(13)$ & $0.0280(15)$ & $0.0337(13)$ & $0.0002(11)$ & $-0.0044(10)$ & $-0.0061(11)$ \\
\hline $\mathrm{C} 27$ & $0.0351(13)$ & $0.0339(16)$ & $0.0340(13)$ & $0.0014(11)$ & $-0.0027(10)$ & $-0.0087(11)$ \\
\hline $\mathrm{C} 28$ & $0.0382(13)$ & $0.0349(16)$ & $0.0377(14)$ & $-0.0058(12)$ & $-0.0014(11)$ & $-0.0093(12)$ \\
\hline $\mathrm{C} 29$ & $0.0327(12)$ & $0.0326(15)$ & $0.0327(13)$ & $-0.0018(11)$ & $0.0012(10)$ & $-0.0071(11)$ \\
\hline $\mathrm{C} 30$ & $0.0273(11)$ & $0.0295(14)$ & $0.0314(12)$ & $0.0026(10)$ & $-0.0020(9)$ & $-0.0086(11)$ \\
\hline C31 & $0.0346(12)$ & $0.0316(15)$ & $0.0326(13)$ & $-0.0004(11)$ & $-0.0004(10)$ & $-0.0064(11)$ \\
\hline $\mathrm{C} 32$ & $0.0323(12)$ & $0.0324(15)$ & $0.0312(13)$ & $0.0036(11)$ & $0.0003(10)$ & $-0.0042(11)$ \\
\hline
\end{tabular}

Geometric parameters $\left(\AA,{ }^{\circ}\right)$

\begin{tabular}{llll}
\hline $\mathrm{O} 1-\mathrm{C} 7$ & $1.319(3)$ & $\mathrm{C} 12-\mathrm{C} 18$ & $1.489(3)$ \\
$\mathrm{O} 1-\mathrm{H} 1$ & $1.04(3)$ & $\mathrm{C} 13-\mathrm{C} 14$ & $1.382(3)$ \\
$\mathrm{O} 2-\mathrm{C} 7$ & $1.225(3)$ & $\mathrm{C} 13-\mathrm{H} 13$ & 0.9500 \\
$\mathrm{O} 3-\mathrm{C} 4$ & $1.363(3)$ & $\mathrm{C} 14-\mathrm{C} 15$ & $1.399(3)$ \\
$\mathrm{O} 3-\mathrm{C} 8$ & $1.435(3)$ & $\mathrm{C} 14-\mathrm{H} 14$ & 0.9500 \\
$\mathrm{O} 4-\mathrm{C} 18$ & $1.322(3)$ & $\mathrm{C} 15-\mathrm{C} 16$ & $1.376(3)$ \\
$\mathrm{O} 4-\mathrm{H} 4$ & $1.00(4)$ & $\mathrm{C} 16-\mathrm{C} 17$ & $1.392(3)$ \\
$\mathrm{O} 5-\mathrm{C} 18$ & $1.214(3)$ & $\mathrm{C} 16-\mathrm{H} 16$ & 0.9500 \\
$\mathrm{O} 6-\mathrm{C} 15$ & $1.362(3)$ & $\mathrm{C} 17-\mathrm{H} 17$ & 0.9500 \\
$\mathrm{O} 6-\mathrm{C} 19$ & $1.438(3)$ & $\mathrm{C} 19-\mathrm{C} 20$ & $1.506(3)$
\end{tabular}




\begin{tabular}{|c|c|c|c|}
\hline $\mathrm{N} 1-\mathrm{C} 27$ & $1.325(4)$ & C19-H19A & 0.9900 \\
\hline $\mathrm{N} 1-\mathrm{C} 23$ & $1.335(4)$ & C19-H19B & 0.9900 \\
\hline $\mathrm{N} 2-\mathrm{C} 28$ & $1.334(3)$ & $\mathrm{C} 20-\mathrm{C} 21$ & $1.518(4)$ \\
\hline $\mathrm{N} 2-\mathrm{C} 32$ & $1.337(3)$ & $\mathrm{C} 20-\mathrm{H} 20 \mathrm{~A}$ & 0.9900 \\
\hline $\mathrm{C} 1-\mathrm{C} 6$ & $1.381(4)$ & $\mathrm{C} 20-\mathrm{H} 20 \mathrm{~B}$ & 0.9900 \\
\hline $\mathrm{C} 1-\mathrm{C} 2$ & $1.398(3)$ & $\mathrm{C} 21-\mathrm{C} 22$ & $1.516(4)$ \\
\hline $\mathrm{C} 1-\mathrm{C} 7$ & $1.475(3)$ & $\mathrm{C} 21-\mathrm{H} 21 \mathrm{~A}$ & 0.9900 \\
\hline $\mathrm{C} 2-\mathrm{C} 3$ & $1.381(3)$ & $\mathrm{C} 21-\mathrm{H} 21 \mathrm{~B}$ & 0.9900 \\
\hline $\mathrm{C} 2-\mathrm{H} 2$ & 0.9500 & $\mathrm{C} 22-\mathrm{H} 22 \mathrm{~A}$ & 0.9800 \\
\hline $\mathrm{C} 3-\mathrm{C} 4$ & $1.390(4)$ & $\mathrm{C} 22-\mathrm{H} 22 \mathrm{~B}$ & 0.9800 \\
\hline $\mathrm{C} 3-\mathrm{H} 3$ & 0.9500 & $\mathrm{C} 22-\mathrm{H} 22 \mathrm{C}$ & 0.9800 \\
\hline $\mathrm{C} 4-\mathrm{C} 5$ & $1.389(3)$ & $\mathrm{C} 23-\mathrm{C} 24$ & $1.382(4)$ \\
\hline $\mathrm{C} 5-\mathrm{C} 6$ & $1.387(3)$ & $\mathrm{C} 23-\mathrm{H} 23$ & 0.9500 \\
\hline $\mathrm{C} 5-\mathrm{H} 5$ & 0.9500 & $\mathrm{C} 24-\mathrm{C} 25$ & $1.389(4)$ \\
\hline $\mathrm{C} 6-\mathrm{H} 6$ & 0.9500 & $\mathrm{C} 24-\mathrm{H} 24$ & 0.9500 \\
\hline $\mathrm{C} 8-\mathrm{C} 9$ & $1.505(3)$ & $\mathrm{C} 25-\mathrm{C} 26$ & $1.386(3)$ \\
\hline $\mathrm{C} 8-\mathrm{H} 8 \mathrm{~A}$ & 0.9900 & $\mathrm{C} 25-\mathrm{C} 30$ & $1.483(3)$ \\
\hline $\mathrm{C} 8-\mathrm{H} 8 \mathrm{~B}$ & 0.9900 & $\mathrm{C} 26-\mathrm{C} 27$ & $1.383(3)$ \\
\hline $\mathrm{C} 9-\mathrm{C} 10$ & $1.504(4)$ & $\mathrm{C} 26-\mathrm{H} 26$ & 0.9500 \\
\hline C9-H9A & 0.9900 & $\mathrm{C} 27-\mathrm{H} 27$ & 0.9500 \\
\hline C9-H9B & 0.9900 & $\mathrm{C} 28-\mathrm{C} 29$ & $1.384(3)$ \\
\hline $\mathrm{C} 10-\mathrm{C} 11$ & $1.523(3)$ & $\mathrm{C} 28-\mathrm{H} 28$ & 0.9500 \\
\hline $\mathrm{C} 10-\mathrm{H} 10 \mathrm{~A}$ & 0.9900 & $\mathrm{C} 29-\mathrm{C} 30$ & $1.394(4)$ \\
\hline $\mathrm{C} 10-\mathrm{H} 10 \mathrm{~B}$ & 0.9900 & $\mathrm{C} 29-\mathrm{H} 29$ & 0.9500 \\
\hline C11-H11A & 0.9800 & $\mathrm{C} 30-\mathrm{C} 31$ & $1.382(3)$ \\
\hline $\mathrm{C} 11-\mathrm{H} 11 \mathrm{~B}$ & 0.9800 & $\mathrm{C} 31-\mathrm{C} 32$ & $1.383(3)$ \\
\hline $\mathrm{C} 11-\mathrm{H} 11 \mathrm{C}$ & 0.9800 & $\mathrm{C} 31-\mathrm{H} 31$ & 0.9500 \\
\hline $\mathrm{C} 12-\mathrm{C} 13$ & $1.387(3)$ & $\mathrm{C} 32-\mathrm{H} 32$ & 0.9500 \\
\hline $\mathrm{C} 12-\mathrm{C} 17$ & $1.390(3)$ & & \\
\hline $\mathrm{C} 7-\mathrm{O} 1-\mathrm{H} 1$ & $107(2)$ & $\mathrm{C} 15-\mathrm{C} 16-\mathrm{C} 17$ & $119.9(2)$ \\
\hline $\mathrm{C} 4-\mathrm{O} 3-\mathrm{C} 8$ & $117.16(19)$ & $\mathrm{C} 15-\mathrm{C} 16-\mathrm{H} 16$ & 120.1 \\
\hline $\mathrm{C} 18-\mathrm{O} 4-\mathrm{H} 4$ & $105(2)$ & $\mathrm{C} 17-\mathrm{C} 16-\mathrm{H} 16$ & 120.1 \\
\hline $\mathrm{C} 15-\mathrm{O} 6-\mathrm{C} 19$ & $117.34(19)$ & $\mathrm{C} 12-\mathrm{C} 17-\mathrm{C} 16$ & $120.5(2)$ \\
\hline $\mathrm{C} 27-\mathrm{N} 1-\mathrm{C} 23$ & $117.5(2)$ & $\mathrm{C} 12-\mathrm{C} 17-\mathrm{H} 17$ & 119.7 \\
\hline $\mathrm{C} 28-\mathrm{N} 2-\mathrm{C} 32$ & $118.2(2)$ & $\mathrm{C} 16-\mathrm{C} 17-\mathrm{H} 17$ & 119.7 \\
\hline $\mathrm{C} 6-\mathrm{C} 1-\mathrm{C} 2$ & $118.9(2)$ & $\mathrm{O} 5-\mathrm{C} 18-\mathrm{O} 4$ & $123.1(2)$ \\
\hline $\mathrm{C} 6-\mathrm{C} 1-\mathrm{C} 7$ & $118.8(2)$ & $\mathrm{O} 5-\mathrm{C} 18-\mathrm{C} 12$ & $123.0(2)$ \\
\hline $\mathrm{C} 2-\mathrm{C} 1-\mathrm{C} 7$ & $122.3(2)$ & $\mathrm{O} 4-\mathrm{C} 18-\mathrm{C} 12$ & $113.9(2)$ \\
\hline $\mathrm{C} 3-\mathrm{C} 2-\mathrm{C} 1$ & $120.5(2)$ & $\mathrm{O} 6-\mathrm{C} 19-\mathrm{C} 20$ & $108.0(2)$ \\
\hline $\mathrm{C} 3-\mathrm{C} 2-\mathrm{H} 2$ & 119.7 & O6- $-\mathrm{C} 19-\mathrm{H} 19 \mathrm{~A}$ & 110.1 \\
\hline $\mathrm{C} 1-\mathrm{C} 2-\mathrm{H} 2$ & 119.7 & $\mathrm{C} 20-\mathrm{C} 19-\mathrm{H} 19 \mathrm{~A}$ & 110.1 \\
\hline $\mathrm{C} 2-\mathrm{C} 3-\mathrm{C} 4$ & $120.0(2)$ & $\mathrm{O} 6-\mathrm{C} 19-\mathrm{H} 19 \mathrm{~B}$ & 110.1 \\
\hline $\mathrm{C} 2-\mathrm{C} 3-\mathrm{H} 3$ & 120.0 & $\mathrm{C} 20-\mathrm{C} 19-\mathrm{H} 19 \mathrm{~B}$ & 110.1 \\
\hline $\mathrm{C} 4-\mathrm{C} 3-\mathrm{H} 3$ & 120.0 & $\mathrm{H} 19 \mathrm{~A}-\mathrm{C} 19-\mathrm{H} 19 \mathrm{~B}$ & 108.4 \\
\hline $\mathrm{O} 3-\mathrm{C} 4-\mathrm{C} 5$ & $123.5(2)$ & $\mathrm{C} 19-\mathrm{C} 20-\mathrm{C} 21$ & $113.9(2)$ \\
\hline $\mathrm{O} 3-\mathrm{C} 4-\mathrm{C} 3$ & $116.6(2)$ & $\mathrm{C} 19-\mathrm{C} 20-\mathrm{H} 20 \mathrm{~A}$ & 108.8 \\
\hline $\mathrm{C} 5-\mathrm{C} 4-\mathrm{C} 3$ & $119.9(2)$ & $\mathrm{C} 21-\mathrm{C} 20-\mathrm{H} 20 \mathrm{~A}$ & 108.8 \\
\hline
\end{tabular}




\begin{tabular}{|c|c|c|c|}
\hline $\mathrm{C} 6-\mathrm{C} 5-\mathrm{C} 4$ & $119.6(2)$ & $\mathrm{C} 19-\mathrm{C} 20-\mathrm{H} 20 \mathrm{~B}$ & 108.8 \\
\hline $\mathrm{C} 6-\mathrm{C} 5-\mathrm{H} 5$ & 120.2 & $\mathrm{C} 21-\mathrm{C} 20-\mathrm{H} 20 \mathrm{~B}$ & 108.8 \\
\hline $\mathrm{C} 4-\mathrm{C} 5-\mathrm{H} 5$ & 120.2 & $\mathrm{H} 20 \mathrm{~A}-\mathrm{C} 20-\mathrm{H} 20 \mathrm{~B}$ & 107.7 \\
\hline $\mathrm{C} 1-\mathrm{C} 6-\mathrm{C} 5$ & $121.1(2)$ & $\mathrm{C} 22-\mathrm{C} 21-\mathrm{C} 20$ & $112.6(2)$ \\
\hline $\mathrm{C} 1-\mathrm{C} 6-\mathrm{H} 6$ & 119.5 & $\mathrm{C} 22-\mathrm{C} 21-\mathrm{H} 21 \mathrm{~A}$ & 109.1 \\
\hline $\mathrm{C} 5-\mathrm{C} 6-\mathrm{H} 6$ & 119.5 & $\mathrm{C} 20-\mathrm{C} 21-\mathrm{H} 21 \mathrm{~A}$ & 109.1 \\
\hline $\mathrm{O} 2-\mathrm{C} 7-\mathrm{O} 1$ & $122.9(2)$ & $\mathrm{C} 22-\mathrm{C} 21-\mathrm{H} 21 \mathrm{~B}$ & 109.1 \\
\hline $\mathrm{O} 2-\mathrm{C} 7-\mathrm{C} 1$ & $122.4(2)$ & $\mathrm{C} 20-\mathrm{C} 21-\mathrm{H} 21 \mathrm{~B}$ & 109.1 \\
\hline $\mathrm{O} 1-\mathrm{C} 7-\mathrm{C} 1$ & $114.7(2)$ & $\mathrm{H} 21 \mathrm{~A}-\mathrm{C} 21-\mathrm{H} 21 \mathrm{~B}$ & 107.8 \\
\hline $\mathrm{O} 3-\mathrm{C} 8-\mathrm{C} 9$ & $108.3(2)$ & $\mathrm{C} 21-\mathrm{C} 22-\mathrm{H} 22 \mathrm{~A}$ & 109.5 \\
\hline $\mathrm{O} 3-\mathrm{C} 8-\mathrm{H} 8 \mathrm{~A}$ & 110.0 & $\mathrm{C} 21-\mathrm{C} 22-\mathrm{H} 22 \mathrm{~B}$ & 109.5 \\
\hline $\mathrm{C} 9-\mathrm{C} 8-\mathrm{H} 8 \mathrm{~A}$ & 110.0 & $\mathrm{H} 22 \mathrm{~A}-\mathrm{C} 22-\mathrm{H} 22 \mathrm{~B}$ & 109.5 \\
\hline $\mathrm{O} 3-\mathrm{C} 8-\mathrm{H} 8 \mathrm{~B}$ & 110.0 & $\mathrm{C} 21-\mathrm{C} 22-\mathrm{H} 22 \mathrm{C}$ & 109.5 \\
\hline $\mathrm{C} 9-\mathrm{C} 8-\mathrm{H} 8 \mathrm{~B}$ & 110.0 & $\mathrm{H} 22 \mathrm{~A}-\mathrm{C} 22-\mathrm{H} 22 \mathrm{C}$ & 109.5 \\
\hline $\mathrm{H} 8 \mathrm{~A}-\mathrm{C} 8-\mathrm{H} 8 \mathrm{~B}$ & 108.4 & $\mathrm{H} 22 \mathrm{~B}-\mathrm{C} 22-\mathrm{H} 22 \mathrm{C}$ & 109.5 \\
\hline $\mathrm{C} 10-\mathrm{C} 9-\mathrm{C} 8$ & $114.8(2)$ & $\mathrm{N} 1-\mathrm{C} 23-\mathrm{C} 24$ & $123.5(3)$ \\
\hline $\mathrm{C} 10-\mathrm{C} 9-\mathrm{H} 9 \mathrm{~A}$ & 108.6 & $\mathrm{~N} 1-\mathrm{C} 23-\mathrm{H} 23$ & 118.3 \\
\hline $\mathrm{C} 8-\mathrm{C} 9-\mathrm{H} 9 \mathrm{~A}$ & 108.6 & $\mathrm{C} 24-\mathrm{C} 23-\mathrm{H} 23$ & 118.3 \\
\hline $\mathrm{C} 10-\mathrm{C} 9-\mathrm{H} 9 \mathrm{~B}$ & 108.6 & $\mathrm{C} 23-\mathrm{C} 24-\mathrm{C} 25$ & $118.5(3)$ \\
\hline $\mathrm{C} 8-\mathrm{C} 9-\mathrm{H} 9 \mathrm{~B}$ & 108.6 & $\mathrm{C} 23-\mathrm{C} 24-\mathrm{H} 24$ & 120.7 \\
\hline $\mathrm{H} 9 \mathrm{~A}-\mathrm{C} 9-\mathrm{H} 9 \mathrm{~B}$ & 107.5 & $\mathrm{C} 25-\mathrm{C} 24-\mathrm{H} 24$ & 120.7 \\
\hline $\mathrm{C} 9-\mathrm{C} 10-\mathrm{C} 11$ & $112.5(2)$ & $\mathrm{C} 26-\mathrm{C} 25-\mathrm{C} 24$ & $118.2(2)$ \\
\hline $\mathrm{C} 9-\mathrm{C} 10-\mathrm{H} 10 \mathrm{~A}$ & 109.1 & $\mathrm{C} 26-\mathrm{C} 25-\mathrm{C} 30$ & $120.8(2)$ \\
\hline $\mathrm{C} 11-\mathrm{C} 10-\mathrm{H} 10 \mathrm{~A}$ & 109.1 & $\mathrm{C} 24-\mathrm{C} 25-\mathrm{C} 30$ & $121.0(2)$ \\
\hline C9- $\mathrm{C} 10-\mathrm{H} 10 \mathrm{~B}$ & 109.1 & $\mathrm{C} 27-\mathrm{C} 26-\mathrm{C} 25$ & $118.9(3)$ \\
\hline $\mathrm{C} 11-\mathrm{C} 10-\mathrm{H} 10 \mathrm{~B}$ & 109.1 & $\mathrm{C} 27-\mathrm{C} 26-\mathrm{H} 26$ & 120.6 \\
\hline $\mathrm{H} 10 \mathrm{~A}-\mathrm{C} 10-\mathrm{H} 10 \mathrm{~B}$ & 107.8 & $\mathrm{C} 25-\mathrm{C} 26-\mathrm{H} 26$ & 120.6 \\
\hline $\mathrm{C} 10-\mathrm{C} 11-\mathrm{H} 11 \mathrm{~A}$ & 109.5 & $\mathrm{~N} 1-\mathrm{C} 27-\mathrm{C} 26$ & $123.4(2)$ \\
\hline $\mathrm{C} 10-\mathrm{C} 11-\mathrm{H} 11 \mathrm{~B}$ & 109.5 & $\mathrm{~N} 1-\mathrm{C} 27-\mathrm{H} 27$ & 118.3 \\
\hline $\mathrm{H} 11 \mathrm{~A}-\mathrm{C} 11-\mathrm{H} 11 \mathrm{~B}$ & 109.5 & $\mathrm{C} 26-\mathrm{C} 27-\mathrm{H} 27$ & 118.3 \\
\hline $\mathrm{C} 10-\mathrm{C} 11-\mathrm{H} 11 \mathrm{C}$ & 109.5 & $\mathrm{~N} 2-\mathrm{C} 28-\mathrm{C} 29$ & $122.9(2)$ \\
\hline $\mathrm{H} 11 \mathrm{~A}-\mathrm{C} 11-\mathrm{H} 11 \mathrm{C}$ & 109.5 & $\mathrm{~N} 2-\mathrm{C} 28-\mathrm{H} 28$ & 118.5 \\
\hline $\mathrm{H} 11 \mathrm{~B}-\mathrm{C} 11-\mathrm{H} 11 \mathrm{C}$ & 109.5 & $\mathrm{C} 29-\mathrm{C} 28-\mathrm{H} 28$ & 118.5 \\
\hline $\mathrm{C} 13-\mathrm{C} 12-\mathrm{C} 17$ & $119.2(2)$ & $\mathrm{C} 28-\mathrm{C} 29-\mathrm{C} 30$ & $118.8(2)$ \\
\hline $\mathrm{C} 13-\mathrm{C} 12-\mathrm{C} 18$ & $123.0(2)$ & $\mathrm{C} 28-\mathrm{C} 29-\mathrm{H} 29$ & 120.6 \\
\hline $\mathrm{C} 17-\mathrm{C} 12-\mathrm{C} 18$ & $117.7(2)$ & $\mathrm{C} 30-\mathrm{C} 29-\mathrm{H} 29$ & 120.6 \\
\hline $\mathrm{C} 14-\mathrm{C} 13-\mathrm{C} 12$ & $120.5(2)$ & $\mathrm{C} 31-\mathrm{C} 30-\mathrm{C} 29$ & $118.0(2)$ \\
\hline $\mathrm{C} 14-\mathrm{C} 13-\mathrm{H} 13$ & 119.7 & $\mathrm{C} 31-\mathrm{C} 30-\mathrm{C} 25$ & $120.5(2)$ \\
\hline $\mathrm{C} 12-\mathrm{C} 13-\mathrm{H} 13$ & 119.7 & $\mathrm{C} 29-\mathrm{C} 30-\mathrm{C} 25$ & $121.5(2)$ \\
\hline $\mathrm{C} 13-\mathrm{C} 14-\mathrm{C} 15$ & $119.8(2)$ & $\mathrm{C} 30-\mathrm{C} 31-\mathrm{C} 32$ & $119.5(2)$ \\
\hline $\mathrm{C} 13-\mathrm{C} 14-\mathrm{H} 14$ & 120.1 & $\mathrm{C} 30-\mathrm{C} 31-\mathrm{H} 31$ & 120.3 \\
\hline $\mathrm{C} 15-\mathrm{C} 14-\mathrm{H} 14$ & 120.1 & $\mathrm{C} 32-\mathrm{C} 31-\mathrm{H} 31$ & 120.3 \\
\hline $\mathrm{O} 6-\mathrm{C} 15-\mathrm{C} 16$ & $124.4(2)$ & $\mathrm{N} 2-\mathrm{C} 32-\mathrm{C} 31$ & $122.5(2)$ \\
\hline $\mathrm{O} 6-\mathrm{C} 15-\mathrm{C} 14$ & $115.6(2)$ & $\mathrm{N} 2-\mathrm{C} 32-\mathrm{H} 32$ & 118.7 \\
\hline $\mathrm{C} 16-\mathrm{C} 15-\mathrm{C} 14$ & $120.0(2)$ & $\mathrm{C} 31-\mathrm{C} 32-\mathrm{H} 32$ & 118.7 \\
\hline $\mathrm{C} 6-\mathrm{C} 1-\mathrm{C} 2-\mathrm{C} 3$ & $1.2(3)$ & $\mathrm{C} 18-\mathrm{C} 12-\mathrm{C} 17-\mathrm{C} 16$ & $179.0(2)$ \\
\hline $\mathrm{C} 7-\mathrm{C} 1-\mathrm{C} 2-\mathrm{C} 3$ & $-177.4(2)$ & $\mathrm{C} 15-\mathrm{C} 16-\mathrm{C} 17-\mathrm{C} 12$ & $1.4(4)$ \\
\hline
\end{tabular}




$\begin{array}{llll}\mathrm{C} 1-\mathrm{C} 2-\mathrm{C} 3-\mathrm{C} 4 & 0.2(4) & \mathrm{C} 13-\mathrm{C} 12-\mathrm{C} 18-\mathrm{O} 5 & 175.6(2) \\ \mathrm{C} 8-\mathrm{O} 3-\mathrm{C} 4-\mathrm{C} 5 & 4.5(3) & \mathrm{C} 17-\mathrm{C} 12-\mathrm{C} 18-\mathrm{O} 5 & -5.2(4) \\ \mathrm{C} 8-\mathrm{O} 3-\mathrm{C} 4-\mathrm{C} 3 & -176.1(2) & \mathrm{C} 13-\mathrm{C} 12-\mathrm{C} 18-\mathrm{O} 4 & -5.6(3) \\ \mathrm{C} 2-\mathrm{C} 3-\mathrm{C} 4-\mathrm{O} 3 & 179.5(2) & \mathrm{C} 17-\mathrm{C} 12-\mathrm{C} 18-\mathrm{O} 4 & 173.5(2) \\ \mathrm{C} 2-\mathrm{C} 3-\mathrm{C} 4-\mathrm{C} 5 & -1.1(4) & \mathrm{C} 15-\mathrm{O} 6-\mathrm{C} 19-\mathrm{C} 20 & 179.9(2) \\ \mathrm{O} 3-\mathrm{C} 4-\mathrm{C} 5-\mathrm{C} 6 & 180.0(2) & \mathrm{O} 6-\mathrm{C} 19-\mathrm{C} 20-\mathrm{C} 21 & -62.8(3) \\ \mathrm{C} 3-\mathrm{C} 4-\mathrm{C} 5-\mathrm{C} 6 & 0.5(4) & \mathrm{C} 19-\mathrm{C} 20-\mathrm{C} 21-\mathrm{C} 22 & -174.4(2) \\ \mathrm{C} 2-\mathrm{C} 1-\mathrm{C} 6-\mathrm{C} 5 & -1.8(3) & \mathrm{C} 27-\mathrm{N} 1-\mathrm{C} 23-\mathrm{C} 24 & 2.1(4) \\ \mathrm{C} 7-\mathrm{C} 1-\mathrm{C} 6-\mathrm{C} 5 & 176.9(2) & \mathrm{N} 1-\mathrm{C} 23-\mathrm{C} 24-\mathrm{C} 25 & -2.6(5) \\ \mathrm{C} 4-\mathrm{C} 5-\mathrm{C} 6-\mathrm{C} 1 & 0.9(4) & \mathrm{C} 23-\mathrm{C} 24-\mathrm{C} 25-\mathrm{C} 26 & 1.0(4) \\ \mathrm{C} 6-\mathrm{C} 1-\mathrm{C} 7-\mathrm{O} 2 & -4.7(4) & \mathrm{C} 23-\mathrm{C} 24-\mathrm{C} 25-\mathrm{C} 30 & 178.9(3) \\ \mathrm{C} 2-\mathrm{C} 1-\mathrm{C} 7-\mathrm{O} 2 & 174.0(2) & \mathrm{C} 24-\mathrm{C} 25-\mathrm{C} 26-\mathrm{C} 27 & 0.8(4) \\ \mathrm{C} 6-\mathrm{C} 1-\mathrm{C} 7-\mathrm{O} 1 & 176.3(2) & \mathrm{C} 30-\mathrm{C} 25-\mathrm{C} 26-\mathrm{C} 27 & -177.1(2) \\ \mathrm{C} 2-\mathrm{C} 1-\mathrm{C} 7-\mathrm{O} 1 & -5.1(3) & \mathrm{C} 23-\mathrm{N} 1-\mathrm{C} 27-\mathrm{C} 26 & -0.2(4) \\ \mathrm{C} 4-\mathrm{O} 3-\mathrm{C} 8-\mathrm{C} 9 & -178.40(19) & \mathrm{C} 25-\mathrm{C} 26-\mathrm{C} 27-\mathrm{N} 1 & -1.3(4) \\ \mathrm{O} 3-\mathrm{C} 8-\mathrm{C} 9-\mathrm{C} 10 & 66.9(3) & \mathrm{C} 32-\mathrm{N} 2-\mathrm{C} 28-\mathrm{C} 29 & 1.6(4) \\ \mathrm{C} 8-\mathrm{C} 9-\mathrm{C} 10-\mathrm{C} 11 & 178.0(2) & \mathrm{N} 2-\mathrm{C} 28-\mathrm{C} 29-\mathrm{C} 30 & -2.3(4) \\ \mathrm{C} 17-\mathrm{C} 12-\mathrm{C} 13-\mathrm{C} 14 & 1.0(4) & \mathrm{C} 28-\mathrm{C} 29-\mathrm{C} 30-\mathrm{C} 31 & 0.5(4) \\ \mathrm{C} 18-\mathrm{C} 12-\mathrm{C} 13-\mathrm{C} 14 & -179.8(2) & \mathrm{C} 28-\mathrm{C} 29-\mathrm{C} 30-\mathrm{C} 25 & 179.6(2) \\ \mathrm{C} 12-\mathrm{C} 13-\mathrm{C} 14-\mathrm{C} 15 & 0.1(4) & \mathrm{C} 26-\mathrm{C} 25-\mathrm{C} 30-\mathrm{C} 31 & 140.9(3) \\ \mathrm{C} 19-\mathrm{O} 6-\mathrm{C} 15-\mathrm{C} 16 & 3.6(4) & \mathrm{C} 24-\mathrm{C} 25-\mathrm{C} 30-\mathrm{C} 31 & -37.0(3) \\ \mathrm{C} 19-\mathrm{O} 6-\mathrm{C} 15-\mathrm{C} 14 & -175.7(2) & \mathrm{C} 26-\mathrm{C} 25-\mathrm{C} 30-\mathrm{C} 29 & -38.2(3) \\ \mathrm{C} 13-\mathrm{C} 14-\mathrm{C} 15-\mathrm{O} 6 & 178.8(2) & \mathrm{C} 24-\mathrm{C} 25-\mathrm{C} 30-\mathrm{C} 29 & 144.0(3) \\ \mathrm{C} 13-\mathrm{C} 14-\mathrm{C} 15-\mathrm{C} 16 & -0.5(4) & \mathrm{C} 29-\mathrm{C} 30-\mathrm{C} 31-\mathrm{C} 32 & 1.8(4) \\ \mathrm{O} 6-\mathrm{C} 15-\mathrm{C} 16-\mathrm{C} 17 & -179.5(2) & \mathrm{C} 25-\mathrm{C} 30-\mathrm{C} 31-\mathrm{C} 32 & -177.3(2) \\ \mathrm{C} 14-\mathrm{C} 15-\mathrm{C} 16-\mathrm{C} 17 & -0.3(4) & \mathrm{C} 28-\mathrm{N} 2-\mathrm{C} 32-\mathrm{C} 31 & 0.9(4) \\ \mathrm{C} 13-\mathrm{C} 12-\mathrm{C} 17-\mathrm{C} 16 & -1.8(4) & \mathrm{C} 30-\mathrm{C} 31-\mathrm{C} 32-\mathrm{N} 2 & -2.6(4)\end{array}$

Hydrogen-bond geometry $\left(A,{ }^{\circ}\right)$

$\mathrm{Cg} 1$ and $\mathrm{Cg} 2$ are the centroids of the $\mathrm{C} 1-\mathrm{C} 6$ and $\mathrm{C} 12-\mathrm{C} 17$ rings, respectively.

\begin{tabular}{lllll}
\hline$D-\mathrm{H} \cdots A$ & $D-\mathrm{H}$ & $\mathrm{H} \cdots A$ & $D \cdots A$ & $D-\mathrm{H} \cdots A$ \\
\hline $\mathrm{O} 1-\mathrm{H} 1 \cdots \mathrm{N} 1$ & $1.04(3)$ & $1.56(3)$ & $2.600(3)$ & $173(3)$ \\
$\mathrm{O} 4-\mathrm{H} 4 \cdots \mathrm{N} 2$ & $1.00(4)$ & $1.64(4)$ & $2.636(3)$ & $172(4)$ \\
$\mathrm{C} 24-\mathrm{H} 24 \cdots \mathrm{O} 5^{\mathrm{i}}$ & 0.95 & 2.47 & $3.408(3)$ & 171 \\
$\mathrm{C} 29-\mathrm{H} 29 \cdots 2^{\mathrm{ii}}$ & 0.95 & 2.53 & $3.456(3)$ & 164 \\
$\mathrm{C} 2-\mathrm{H} 2 \cdots C g 2^{i i i}$ & 0.95 & 2.98 & $3.754(3)$ & 139 \\
$\mathrm{C} 8-\mathrm{H} 8 B \cdots C g 2^{\mathrm{iv}}$ & 0.99 & 2.68 & $3.518(3)$ & 143 \\
$\mathrm{C} 19-\mathrm{H} 19 B \cdots C g 1^{\mathrm{v}}$ & 0.99 & 2.77 & $3.586(3)$ & 140 \\
\hline
\end{tabular}

Symmetry codes: (i) $x, y-1, z$; (ii) $x, y+1, z$; (iii) $-x,-y+1,-z$; (iv) $-x+1,-y,-z$; (v) $-x+1,-y+1,-z$. 